\title{
Kleine-Levin syndrome is associated with birth difficulties and genetic variants in the TRANK1 gene loci
}

\author{
Aditya Ambati ${ }^{a, 1} \oplus$, Ryan Hillary ${ }^{a, 1}$, Smaranda Leu-Semenescu ${ }^{b, 2} \odot$, Hanna M. Ollila ${ }^{a, 2}$, Ling Lin ${ }^{a}(0$, \\ Emmanuel H. During ${ }^{\text {c,d }} \odot$, Neal Farber ${ }^{e} \odot$, Thomas J. Rico ${ }^{\mathrm{a}}{ }^{\circ}$, Juliette Faraco ${ }^{\mathrm{a}}$, Eileen Leary ${ }^{\mathrm{a}} \odot$,

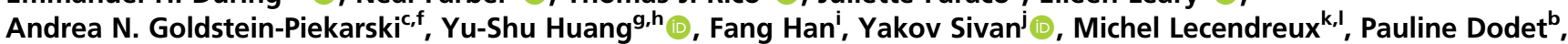 \\ Makoto Honda $^{\mathrm{m}}$, Natan Gadoth ${ }^{\mathrm{n}, o}$, Sona Nevsimalova ${ }^{\mathrm{p}}{ }^{\infty}$, Fabio Pizza $^{\mathrm{q}, \mathrm{r}}$, Takashi Kanbayashi ${ }^{\mathrm{s}}$, Rosa Peraita-Adrados ${ }^{\mathrm{t}}{ }^{\circ}$,

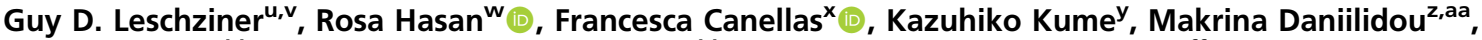 \\ Patrice Bourgin $^{\text {bb }}{ }^{(0}$, David Rye ${ }^{c c}$, José L. Vicario ${ }^{\text {dd }}$, Birgit Hogl ${ }^{\text {ee }}$, Seung Chul Hong ${ }^{\text {ff }}$, Guiseppe Plazzi ${ }^{\text {,r }}$, Geert Mayer ${ }^{\text {gg }}$, \\ Anne Marie Landtblom ${ }^{z, a a}$, Yves Dauvilliers ${ }^{\text {hh,ii }}$, Isabelle Arnulf ${ }^{b, 1}$, and Emmanuel Jean-Marie Mignot ${ }^{\text {a, }, 3}$
}

\begin{abstract}
${ }^{a}$ Center for Sleep Sciences and Medicine, Stanford University School of Medicine, Palo Alto, CA 94304; bSleep Disorders, Pitié-Salpêtrière Hospital, Assistance Publique-Hôpitaux de Paris-Sorbonne, National Reference Center for Narcolepsy, Idiopathic Hypersomnia and Kleine-Levin Syndrome, Sorbonne University, Institut Hospitalo-Universitaire A Institut du Cerveau et de la Moelle, F-75013 Paris, France; 'Department of Psychiatry \& Behavioral Sciences, Stanford University, Stanford, CA 94305; ${ }^{d}$ Department of Neurology \& Neurological Sciences, Stanford University, Stanford, CA 94305; ${ }^{\text {KKleine-Levin }}$ Syndrome Foundation, Boston, MA 02468; 'Sierra-Pacific Mental Illness Research, Education, and Clinical Center, Veterans Affairs Palo Alto Health Care System, Palo Alto, CA 94304; ${ }^{9}$ Department of Child Psychiatry, Chang Gung Memorial Hospital and University, Taoyuan 33305, Taiwan; ${ }^{\text {h}}$ Sleep Center, Chang Gung Memorial Hospital and University, Taoyuan, 33305, Taiwan; 'Department of Pulmonary Medicine, Peking University People's Hospital, Beijing 100044, China; 'Safra Children's Hospital, Sheba Medical Center, Sackler Faculty of Medicine, Tel Aviv University, 52621 Tel Aviv, Israel; ${ }^{k}$ Pediatric Sleep Center, Hospital Robert Debre, France Center for Narcolepsy and Idiopathic Hypersomnia, 75019 Paris, France; 'National Reference Center, Hospital Robert Debre, France Center for Narcolepsy and Idiopathic Hypersomnia, 75019 Paris, France; ${ }^{m}$ Sleep Disorders Project, Department of Psychiatry and Behavioral Sciences, Tokyo Metropolitan Institute of Medical Science, Tokyo 156-8506, Japan; ${ }^{n}$ Department of Neurology, Maynei Hayeshua Medical Center, 5154475 Bnei Barak, Israel; ${ }^{\circ}$ The Sackler Faculty of Medicine, Tel-Aviv University, 6997801 Tel Aviv, Israel; ${ }^{\circ}$ Department of Neurology, 1st Faculty of Medicine, General Teaching Hospital, Charles University, Prague 11636 , Czech Republic; ${ }^{9}$ Department of Biomedical and Neuromotor Sciences, University of Bologna, 40139 Bologna, Italy; 'Istituto di Ricovero e Cura a Carattere Scientifico - IRCCS Institute of Neurological Sciences, 40139 Bologna, Italy; ${ }^{5}$ International Institute for Integrative Sleep Medicine, University of Tsukuba, Tsukuba 305-0005, Japan; ' ${ }^{\mathrm{H}}$ ospital Universitario e Instituto de Investigación Gregorio Marañón, Universidad Complutense de Madrid, 28040 Madrid, Spain; "'Sleep Disorders Centre, Guy's Hospital, SE1 9RT London, United Kingdom; ${ }^{V}$ Institute of Psychiatry, Psychology and Neuroscience, King's College London, SE5 8AF London, United Kingdom; ' 'Institute of Psychiatry, Hospital das Clinicas, Faculty of Medicine, University of Sao Paulo, Sao Paulo 05403-010, Brazil; ${ }^{\mathrm{X} F u n d a c i o ́ ~ I n s t i t u t ~ d ' I n v e s t i g a c i o ́ ~ S a n i t a ̀ r i a ~ I l l e s ~ B a l e a r s, ~ H o s p i t a l ~ U n i v e r s i t a r i ~ S o n ~ E s p a s e s, ~}$ 07120 Palma, Spain; ${ }^{y}$ Department of Neuropharmacology, Nagoya City University, Nagoya 467-8601, Japan; ${ }^{2}$ Department of Neuroscience, Uppsala University, Uppsala 752 36, Sweden; ${ }^{a}$ Department of Biomedical and Clinical Sciences, Linköping University, Linköping 581 83, Sweden; bb Sleep Disorders Center, Hôpitaux Universitaires de Strasbourg, 67091 Strasbourg, France; “cDepartment of Neurology, Emory University, Atlanta, GA 30322;

${ }^{\mathrm{dd}}$ Histocompatibility Department, Blood Center of the Community of Madrid, 28032 Madrid, Spain; ${ }^{e}$ Department of Neurology, Innsbruck Medical University, 6020 Innsbruck, Austria; ff Department of Neuropsychiatry, College of Medicine, St. Vincent's Hospital, Catholic University of Korea, Seoul 16247,

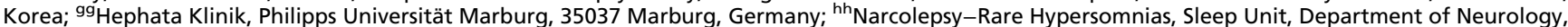
National Reference Centre for Orphan Diseases, Centre Hospitalier Universitaire de Montpellier, Universitaire de Montpellier, 34000 Montpellier, France; and iinstitute for Neurosciences of Montpellier, Universitaire de Montpellier, INSERM, 34000 Montpellier, France
\end{abstract}

Contributed by Emmanuel Jean-Marie Mignot, August 26, 2020 (sent for review March 31, 2020; reviewed by Nelson B. Freimer and James A. Knowles)

Kleine-Levin syndrome (KLS) is a rare disorder characterized by severe episodic hypersomnia, with cognitive impairment accompanied by apathy or disinhibition. Pathophysiology is unknown, although imaging studies indicate decreased activity in hypothalamic/thalamic areas during episodes. Familial occurrence is increased, and risk is associated with reports of a difficult birth. We conducted a worldwide case-control genome-wide association study in $673 \mathrm{KLS}$ cases collected over $14 \mathrm{y}$, and ethnically matched 15,341 control individuals. We found a strong genome-wide significant association (rs71947865, Odds Ratio $[O R]=1.48, P=8.6 \times 10^{-9}$ ) within the $3^{\prime}$ region of TRANK1 gene locus, previously associated with bipolar disorder and schizophrenia. Strikingly, KLS cases with rs71947865 variant had significantly increased reports of a difficult birth. As perinatal outcomes have dramatically improved over the last $40 \mathrm{y}$, we further stratified our sample by birth years and found that recent cases had a significantly reduced rs71947865 association. While the rs71947865 association did not replicate in the entire follow-up sample of 171 KLS cases, rs71947865 was significantly associated with KLS in the subset follow-up sample of 59 KLS cases who reported birth difficulties (OR = $1.54, P=0.01)$. Genetic liability of KLS as explained by polygenic risk scores was increased (pseudo $R^{2}=0.15 ; P<2.0 \times 10^{-22}$ at $P=0.5$ threshold) in the follow-up sample. Pathway analysis of genetic associations identified enrichment of circadian regulation pathway genes in KLS cases. Our results suggest links between KLS, circadian regulation, and bipolar disorder, and indicate that the TRANK1 polymorphisms in conjunction with reported birth difficulties may predispose to KLS.

Kleine-Levin syndrome | hypersomnia | bipolar disorder |

birth difficulties | GWAS
7 leine-Levin syndrome (KLS) was identified as a unique disease entity almost a century ago $(1,2)$. It affects primarily male teenagers and is characterized by unpredictable periods (median length of $\sim 10 \mathrm{~d}$ ) of intense hypersomnia ( $\geq 18 \mathrm{~h}$ of sleep per day), occasionally associated with megaphagia and sexual disinhibition. More recent descriptions in large case series have confirmed a stereotypic appearance of abrupt onset episodes in mostly male (66 to $75 \%$ ) patients and association of severe hypersomniac episodes with cognitive and behavioral disturbances

Author contributions: A.A., R. Hillary, S.L.-S., E.H.D., N.F., T.J.R., J.F., E.L., Y.-S.H., F.H., Y.S., M.L., P.D., M.H., N.G., S.N., F.P., T.K., R.P.-A., G.D.L., R. Hasan, F.C., K.K., M.D., P.B., D.R., J.L.V., B.H., S.C.H., G.P., G.M., A.M.L., Y.D., I.A., and E.J.-M.M. performed research; A.A., R. Hillary, S.L.-S., H.M.O., and L.L. contributed new reagents/analytic tools; A.A., R. Hillary, S.L.-S., H.M.O., L.L., N.F., I.A., and E.J.-M.M. analyzed data; A.A., R. Hillary, S.L.-S., A.N.G.-P., I.A., and E.J.-M.M. wrote the paper; L.L., E.H.D., N.F., T.J.R., E.L., Y.-S.H., F.H., Y.S., M.L., P.D., M.H., N.G., S.N., F.P., T.K., R.P.-A., G.D.L., R. Hasan, F.C., K.K., M.D., P.B., D.R., J.L.V., B.H., S.C.H., G.P., G.M., A.M.L., Y.D., I.A., and E.J.-M.M performed patient recruitment; A.N.G.-P. provided input on manuscript; and E.J.-M.M. provided study supervision.

Reviewers: N.B.F., Center for Neurobehavioral Genetics, University of California, Los Angeles; and J.A.K., SUNY Downstate Medical Center.

The authors declare no competing interest.

Published under the PNAS license.

${ }^{1}$ A.A., R.H., I.A., and E.J.-M.M. contributed equally to this work.

${ }^{2}$ S.L.S. and H.M.O. contributed equally to this work.

${ }^{3}$ To whom correspondence may be addressed. Email: mignot@stanford.edu.

This article contains supporting information online at https://www.pnas.org/lookup/suppl/ doi:10.1073/pnas.2005753118/-/DCSupplemental.

Published March 18, 2021. 
Significance

Genetic markers in TRANK1 gene and its vicinity have been weakly associated with bipolar disorder and schizophrenia (10\% increased risk). We found that the same polymorphisms are associated with Kleine-Levin syndrome $(50 \%$ increased risk), a rare sleep disorder characterized by recurrent episodes of severe hypersomnia and cognitive abnormalities. Response to lithium treatment is suggestive of a pathophysiological overlap between KLS and bipolar disorder. The study also shows that variants in the TRANK1 gene region may predispose to KLS when patients have had a difficult birth, suggesting that TRANK1 gene region modulates newborns' response to brain injury, with consequences for mental and neurological health in adulthood. Another possibility may be that the polymorphism impacts birth and KLS.

(mental slowness, confusion, apathy, derealization, altered perception, and disinhibited behavior). Remarkably, there is a complete absence of the symptoms between episodes, and a generally favorable evolution, with spontaneous disappearance of episodes after one to two decades (3-6). KLS episodes are dramatic in appearance, and patients are fully incapacitated. Electroencephalogram (EEG) studies occasionally reveal nonspecific EEG slowing during episodes. Further, lithium has been shown to reduce episodes in about one-third of cases $(4,7)$. Episodes may be associated with flu-like symptoms at the onset, with seasonal winter occurrence suggested by some (8).

Although most cases of KLS are sporadic, familial occurrence ( 3 to $8 \%$ of relatives) has been reported in studies $(5,9)$, and there are reports of concordant twin pairs $(9,10)$ which, considering the rarity of the condition (a few cases per million individuals) (11), suggests genetic or shared environmental effects. Early studies had suggested a human leukocyte antigen (HLA) association suggestive of an autoimmune mechanism (12), but this was not confirmed in larger samples $(3,8,13)$. Further, karyotyping was normal in 112 patients, except for one patient with sporadic KLS who had a duplication in Xp22.31 (13).

This study reports genetic associations in a discovery cohort of 673 KLS cases with attempted replication in 171 cases across the world. Additionally, targeted exome sequencing in 32 cases derived from 16 multiplex families (9) was conducted. A KLS genome-wide association study (GWAS) found a strong signal in the TRANK1 region (rs71947865), similar to that reported in bipolar disorder and schizophrenia. In these latter disorders, the TRANK1 association variably maps to rs9834970 in Caucasians or to rs3732386 in multiethnic populations, both singlenucleotide polymorphisms (SNPs) located immediately $3^{\prime}$ of the TRANK1 gene (14-16). Strikingly, the TRANK1 signal was mostly present in KLS patients reporting birth difficulties and decreased over time (defined by birth year), paralleling wellestablished improved perinatal care. Birth difficulties (and maternal infections) have also long been associated with increased risk of bipolar disorder and schizophrenia (17-29). These results indicate possible gene $\times$ environment interactions in KLS, with possibly similar effects in other TRANK1-associated disorders such as bipolar disorder and schizophrenia.

\section{Results}

GWAS of KLS. A worldwide multiethnic cohort of 673 KLS cases, the largest sample of KLS cases ever to be analyzed, was collected between the years of 2003 and 2017 and compared to 15,341 matched controls (the "discovery cohort"; see Dataset S1 for sample construction, and see SI Appendix, Fig. S1 for corresponding principal component analysis [PCA] plots). Genetic association analyses (genomic inflation $\lambda \mathrm{GC}=1.06$ ) found a genome-wide significant effect (Fig. 1) with no heterogeneity across ethnic groups, with more than 20 tightly linked SNPs encompassing a 35-kb region of human chromosome 3 in the 3 region of the tetratricopeptide repeat and ankyrin repeatcontaining protein (TRANK1) gene. The TRANK1 gene was observed to have the highest expression in the fallopian tube, closely followed by the cerebellum (GTEx v8) (see Table 1 and

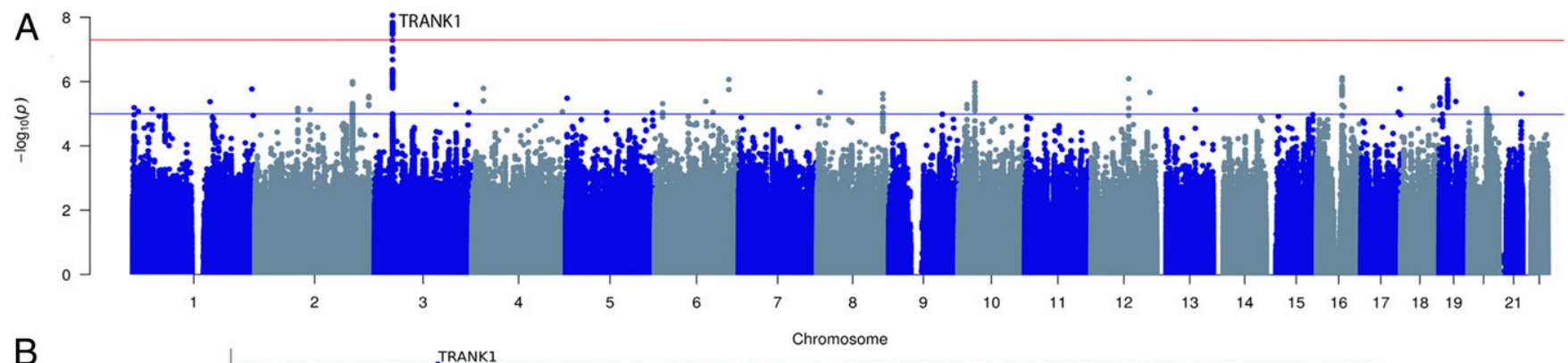

B

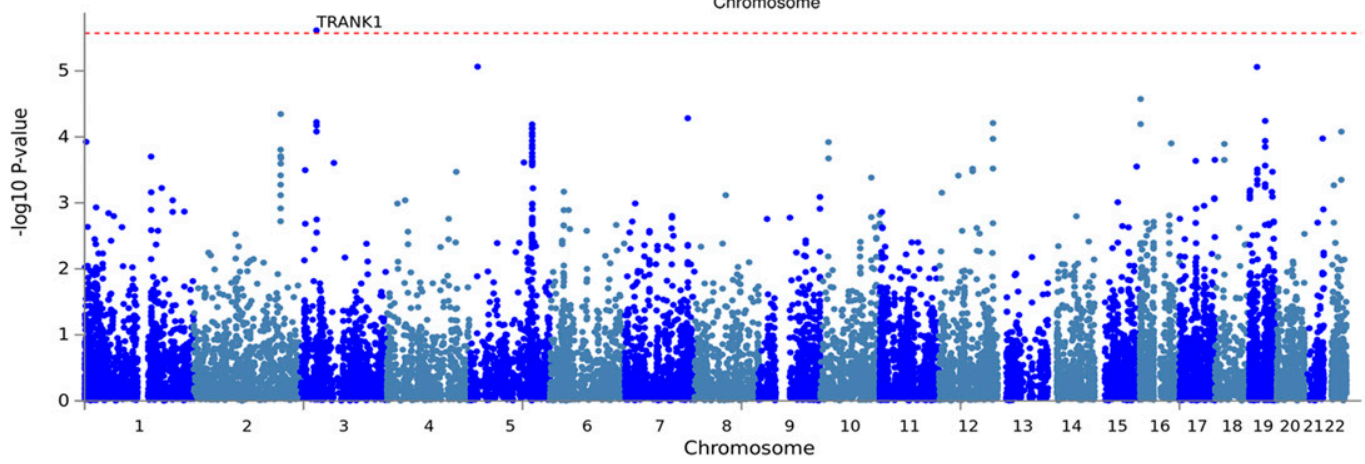

Fig. 1. Multiethnic GWAS in $673 \mathrm{KLS}$ cases and 15,431 control individuals. $(A)$ Manhattan plot of the metaanalysis association (discovery cohort) results from SNP-based GWAS study. The $x$ axis shows genomic location by chromosome, and the $y$ axis shows -log10 $P$ values. Red horizontal line indicates genome-wide significant $P$ value threshold of $5 \times 10^{-8}$. Individual loci are annotated. $(B)$ Manhattan plot of gene-based association test association results. SNPs were mapped to 18,247 protein coding genes (distance 0 ). Genome-wide significance (red dashed line) was defined at $P=0.05 / 18,247=2.74 \times 10^{-6}$. 
Fig. 2 for zoom plot, and see Dataset S2, where all variants in this locus are listed). The most significant association was observed at rs71947865 $\left(\mathrm{OR}=1.47, P=8.6 \times 10^{-9}\right.$, presence of $\mathrm{G}$ versus GGGAGCCA), although the highest OR was observed at rs6789885 $\left(\mathrm{OR}=1.53, P=8.9 \times 10^{-8}, \mathrm{~T}\right.$ versus C; Dataset S2). This TRANK1 region SNP rs71947865 is in absolute linkage disequilibrium (LD) with rs3732386 $\left(r^{2}=1\right)$, an SNP associated with bipolar disorder and schizophrenia in multiethnic samples (Dataset S3).

The LD block contains several eQTL (expression quantitative trait loci) effects affecting numerous genes such as TRANK1, LRRFIP2, MLH1, GOLGA4, DCLK3, STAC, and long noncoding $R N A$ RP11-640L9.2. The SNP rs11129735 (KLS discovery GWAS $P=9.9 \times 10^{-6}$; LD $r^{2}=0.4$ with our top GWAS significant variants) had the highest coloc (CLPP) probability ( $>0.95$ causal probability) using eCAVIAR, with the expression of $L R R F I P 2$ in the thyroid, DCLK3 in skin and brain frontal cortex, C3orf35 in testis, MLH1 in breast tissue, TRANK1 in the spinal cord, and EPM2AIP1 in the hypothalamus (GTEx v7). Querying the GTEx v8 database (30), we found our lead SNP rs71947865 variant to be a strong eQTL for LRRFIP2 gene expression in the thyroid $\left(P=8.2 \times 10^{-20}\right)$ and TRANK1 expression in breast tissue and whole blood. The rs71947865 also had multiple effects on $L R R F I P 2$ expression in various tissues $\left(P<10^{-6}\right.$, GTEx portal v8). LRRFIP2 together with $M L H 1$ and GOLGA4 are highly expressed in placenta and differentially expressed in placentae from complicated compared to normal pregnancies $(31,32)$. The tissue expression for most of these genes is widespread in the brain and poorly characterized. For the reason of convenience, we further called this effect "TRANK1-region polymorphisms" or "TRANK1 effect," although data to date do not allow genetic attribution of the observed effects to secondary changes in TRANK1 gene expression.
Association of Symptoms with Polymorphisms. Clinical data collected from 592 of 673 KLS patients from the discovery cohort were next scrutinized for genotype effects. Our a priori hypothesis for testing was to explore whether TRANK1 polymorphisms were associated with more depressive symptoms, considering lithium responsiveness in the syndrome, and/or with a differential effect on birth difficulties, a robust finding in our prior study (3). Other variables were compared as exploratory analyses. TRANK1 rs71947865 (G) genotype was most significantly associated with reports of birth difficulties $(\mathrm{OR}=1.65 ; P<0.005$ Fisher's exact test of allelic counts) and developmental delays. In addition, patients with TRANK1 rs71947865 (G) have more severe cognitive symptoms, a longer duration of first episode, a more frequent family history of KLS, an older age of onset, and more behavioral disinhibition and altered speech during episodes, generally suggesting more severe disease (Table 2; full list of variables tested are provided in Dataset S4). Types of birth difficulties are summarized in Dataset S5. In a further analysis of the association of clinical variables with the genotype dose of the TRANK1 deletion, we adjusted for reports of birth difficulties. We noted that cognitive signs, age of onset, and longer first episode duration were significantly $(P<0.05)$ increased in KLS cases with TRANK1 deletion allele, while there was a trend to association of TRANK1 deletion allele with current depression in KLS cases (Table 3 ).

TRANK1 genotyping was conducted in 195 controls with birth difficulty data available. These controls were from the KLS case control study reported by Arnulf et al. (3), with further addition of controls (friend or spouse of KLS patient) over more recent years. In the control sample, 22 subjects reported birth difficulties and, unlike in the KLS cases, we found no significant association of birth difficulties with TRANK1 rs71947865 genotype

Table 1. Top variants in TRANK1 region from chromosome 3 associated with KLS in worldwide discovery cohort GWAS metaanalysis in 673 KLS cases and 15,341 control individuals and in a follow-up replication cohort of $171 \mathrm{KLS}$ cases and 1,956 controls

\begin{tabular}{|c|c|c|c|c|c|c|c|c|c|}
\hline \multirow[b]{2}{*}{ SNP } & \multirow[b]{2}{*}{ BP } & \multirow[b]{2}{*}{$A / B$} & \multicolumn{3}{|c|}{$\begin{array}{l}\text { GWAS metaanalysis, } \\
\qquad n=673\end{array}$} & \multicolumn{2}{|c|}{$\begin{array}{c}\text { Follow-up } \\
\text { samples, } \\
n=171\end{array}$} & \multicolumn{2}{|c|}{$\begin{array}{c}\text { Combined, } \\
n=844\end{array}$} \\
\hline & & & Freq. B & OR & $P$ value & OR & $P$ value & OR & $P$ value \\
\hline rs71947865 & 36877798 & GGGAGCCA/G & 0.29 & 1.48 & $8.61 \times 10^{-09}$ & 1.17 & 0.19 & 1.40 & $3.51 \times 10^{-8}$ \\
\hline rs3732385 & 36872193 & C/A & 0.30 & 1.47 & $1.44 \times 10^{-8}$ & 1.17 & 0.18 & 1.39 & $5.00 \times 10^{-8}$ \\
\hline rs3732384 & 36872224 & $\mathrm{~T} / \mathrm{C}$ & 0.30 & 1.47 & $1.44 \times 10^{-8}$ & 1.17 & 0.18 & 1.39 & $5.00 \times 10^{-8}$ \\
\hline rs7652637 & 36871507 & $\mathrm{~T} / \mathrm{C}$ & 0.30 & 1.47 & $1.47 \times 10^{-8}$ & 1.18 & 0.18 & 1.39 & $5.06 \times 10^{-8}$ \\
\hline rs9821223 & 36883369 & $\mathrm{~T} / \mathrm{C}$ & 0.29 & 1.47 & $1.5 \times 10^{-8}$ & 1.11 & 0.39 & 1.38 & $1.67 \times 10^{-7}$ \\
\hline rs9811916 & 36878086 & $A / G$ & 0.30 & 1.47 & $1.63 \times 10^{-8}$ & 1.17 & 0.19 & 1.39 & $5.78 \times 10^{-8}$ \\
\hline rs4678909 & 36878563 & $\mathrm{~A} / \mathrm{G}$ & 0.30 & 1.47 & $1.73 \times 10^{-8}$ & 1.16 & 0.21 & 1.39 & $7.42 \times 10^{-8}$ \\
\hline rs6771655 & 36884370 & C/A & 0.29 & 1.47 & $1.74 \times 10^{-8}$ & 1.11 & 0.39 & 1.38 & $1.91 \times 10^{-7}$ \\
\hline rs9882911 & 36885392 & $\mathrm{~T} / \mathrm{C}$ & 0.29 & 1.47 & $1.78 \times 10^{-8}$ & 1.11 & 0.39 & 1.38 & $1.96 \times 10^{-7}$ \\
\hline rs9863798 & 36885960 & $\mathrm{C} / \mathrm{T}$ & 0.29 & 1.47 & $1.99 \times 10^{-8}$ & 1.11 & 0.39 & 1.37 & $2.16 \times 10^{-7}$ \\
\hline rs4678910 & 36887973 & $\mathrm{C} / \mathrm{G}$ & 0.29 & 1.47 & $2.03 \times 10^{-8}$ & 1.11 & 0.41 & 1.37 & $2.38 \times 10^{-7}$ \\
\hline rs4678911 & 36888064 & $\mathrm{G} / \mathrm{T}$ & 0.29 & 1.47 & $2.09 \times 10^{-8}$ & 1.11 & 0.40 & 1.37 & $2.29 \times 10^{-7}$ \\
\hline rs9819304 & 36888172 & $T / G$ & 0.29 & 1.47 & $2.1 \times 10^{-8}$ & 1.11 & 0.40 & 1.37 & $2.30 \times 10^{-7}$ \\
\hline rs17807744 & 36894048 & $\mathrm{C} / \mathrm{T}$ & 0.29 & 1.46 & $2.18 \times 10^{-8}$ & 1.10 & 0.45 & 1.37 & $2.92 \times 10^{-7}$ \\
\hline rs4234258 & 36878970 & $A / G$ & 0.30 & 1.46 & $2.26 \times 10^{-8}$ & 1.16 & 0.23 & 1.38 & $9.95 \times 10^{-8}$ \\
\hline rs3732386 & 36871993 & $\mathrm{C} / \mathrm{T}$ & 0.30 & 1.46 & $2.26 \times 10^{-8}$ & 1.17 & 0.18 & 1.39 & $7.16 \times 10^{-8}$ \\
\hline rs6550435 & 36864489 & $\mathrm{~T} / \mathrm{G}$ & 0.29 & 1.47 & $2.37 \times 10^{-8}$ & 1.17 & 0.20 & 1.39 & $8.62 \times 10^{-8}$ \\
\hline rs17195098 & 36893965 & $\mathrm{C} / \mathrm{T}$ & 0.29 & 1.46 & $2.83 \times 10^{-8}$ & 1.10 & 0.45 & 1.36 & $3.63 \times 10^{-7}$ \\
\hline rs9883001 & 36894579 & $\mathrm{C} / \mathrm{T}$ & 0.29 & 1.46 & $2.9 \times 10^{-8}$ & 1.10 & 0.45 & 1.36 & $3.70 \times 10^{-7}$ \\
\hline rs4678912 & 36894646 & $\mathrm{~A} / \mathrm{T}$ & 0.29 & 1.46 & $2.91 \times 10^{-8}$ & 1.10 & 0.45 & 1.36 & $3.71 \times 10^{-7}$ \\
\hline rs1532965 & 36861196 & T/G & 0.30 & 1.46 & $2.92 \times 10^{-8}$ & 1.16 & 0.22 & 1.38 & $1.20 \times 10^{-7}$ \\
\hline rs4678913 & 36895741 & $\mathrm{C} / \mathrm{G}$ & 0.29 & 1.46 & $3.05 \times 10^{-8}$ & 1.10 & 0.45 & 1.36 & $3.93 \times 10^{-7}$ \\
\hline rs11706780 & 36896235 & $\mathrm{C} / \mathrm{T}$ & 0.29 & 1.46 & $3.12 \times 10^{-8}$ & 1.09 & 0.46 & 1.36 & $4.04 \times 10^{-7}$ \\
\hline rs4624519 & 36862980 & $\mathrm{C} / \mathrm{T}$ & 0.30 & 1.45 & $3.39 \times 10^{-8}$ & 1.16 & 0.22 & 1.38 & $1.36 \times 10^{-7}$ \\
\hline
\end{tabular}

Effect allele is B; genomic coordinates are in hg19/GRCh37; Freq. B is the frequency of the minor allele B. 


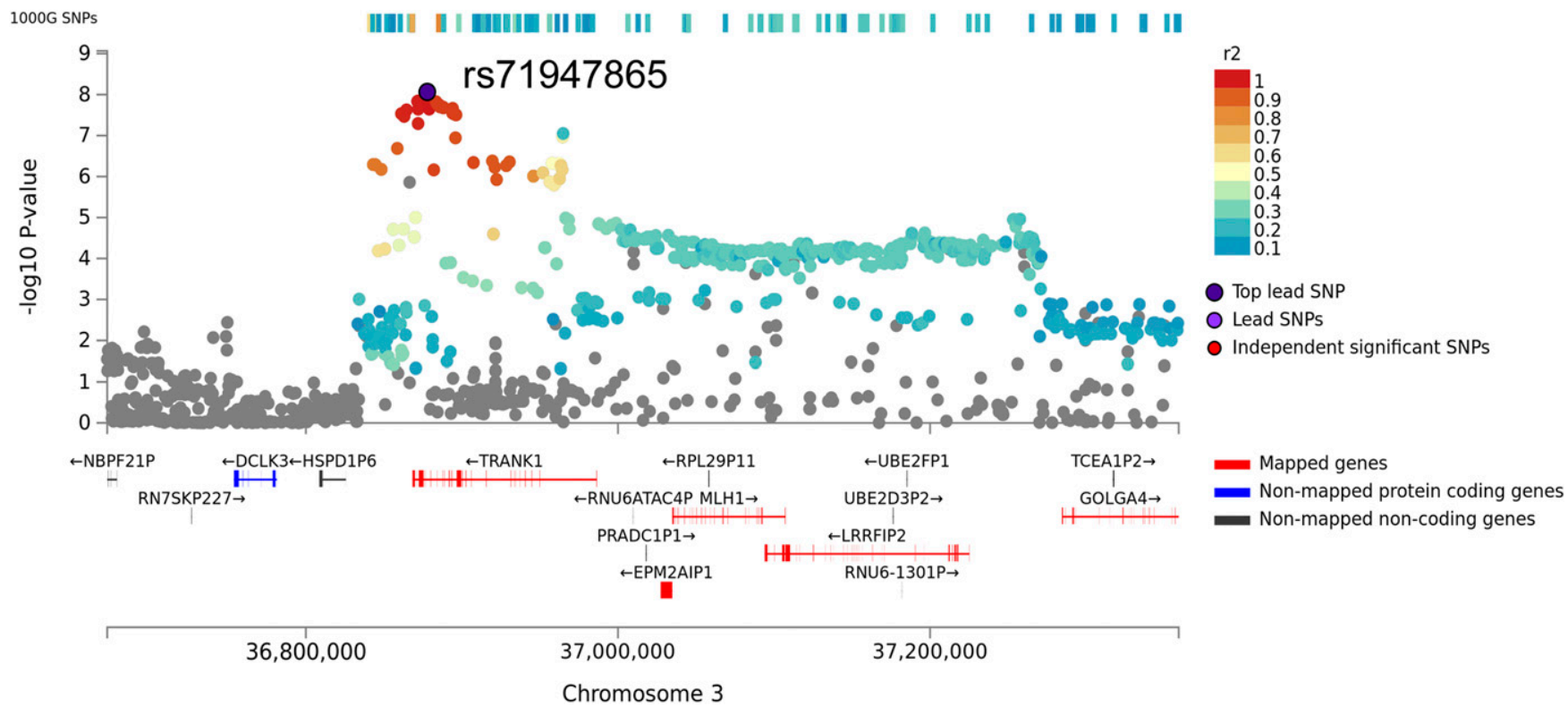

Fig. 2. Regional association plot of the GWA significance of chromosome 3 flanked by rs76652637 and rs 11706780 in the $3^{\prime}$ region of TRANK1. Highest significance was observed at rs71947865 (OR $=1.47, P=8.6 \times 10^{-9}$, presence of G versus GGGAGCCA). The variants are color coded by the magnitude of LD with the rs71947865.

$(P=0.5, \mathrm{OR}=1.05)$. To formally test for an interaction effect of TRANK1 genotype on birth difficulties in KLS versus controls, we used the combined KLS cases-control samples matched by PCA. Whereas both birth difficulties and TRANK1 rs71947865 had a significant effect on KLS risk $(P<0.05)$, the interaction term of TRANK1 rs71947865 and presence of birth difficulties did not reach statistical significance. Note that the sample of controls with clinical (birth difficulty) data available is small and, although it was also used in the GWAS as a control, most of the controls used for GWAS were drawn from the Genetic Epidemiology Research on Aging (GERA) (33) cohort and/or other controls obtained through other studies from the same countries, which come from similar countries and collaborators.
The TRANK1 Association Is Birth Year Dependent. As indicated above, our analysis revealed a significant association of the TRANK1 variant with birth difficulties in KLS patients but not controls. As perinatal care has improved over recent decades (34-39) (https:// www.cdc.gov/nchs/data/nvsr/nvsr56/nvsr56_10.pdf; https://mchb.hrsa. gov/sites/default/files/mchb/Data/Chartbooks/child-health-2014.pdf), most notably, in the 1990-2000s, we asked whether the year of birth was an essential factor correlated to KLS in conjunction with TRANKI rs71947865 variant. A possible birth year-dependent TRANK1 interaction is also supported by the recent largest bipolar disorder GWAS ever published, where it was noteworthy that the strong TRANK1 SNP association found in the discovery sample in bipolar disorder cases did not replicate in a new, presumably more recent,

Table 2. TRANK1 deletion (rs71947865) and its univariate association with KLS clinical variables

\begin{tabular}{|c|c|c|c|c|c|c|c|c|c|}
\hline \multirow[b]{2}{*}{ Clinical variable } & \multicolumn{2}{|c|}{ Model1 } & \multicolumn{2}{|c|}{ Model2 } & \multicolumn{2}{|c|}{ Fisher's } & \multirow[b]{2}{*}{$\mathrm{BB}(n=81)$} & \multirow[b]{2}{*}{$\mathrm{AB}(n=276)$} & \multirow[b]{2}{*}{$\mathrm{AA}(n=235)$} \\
\hline & $P$ & beta & $P$ & Beta & $P$ & Allelic OR & & & \\
\hline Personal history of birth difficulties & 0.0021 & 0.50 & 0.0147 & 0.41 & 0.0021 & 1.66 & $32.4 \%(22 / 68)$ & $21.9 \%(48 / 219)$ & $14.8 \%(27 / 183)$ \\
\hline $\begin{array}{l}\text { Severe cognitive symptoms during } \\
\text { episodes }\end{array}$ & 0.011 & 0.82 & 0.0505 & 0.65 & 0.0089 & 2.24 & $97.1 \%(67 / 69)$ & $96.3 \%(233 / 242)$ & $90.4 \%(179 / 198)$ \\
\hline Family history of KLS & 0.0427 & 0.27 & 0.614 & -0.07 & 0.0484 & 1.31 & $53.1 \%(34 / 64)$ & $61.7 \%(145 / 235)$ & $46.6 \%(97 / 208)$ \\
\hline Behavioral disinhibition & 0.0453 & 0.28 & 0.287 & 0.15 & 0.0475 & 1.34 & $69.6 \%(48 / 69)$ & $72.4 \%(163 / 225)$ & $60.7 \%(116 / 191)$ \\
\hline Altered speech during episodes & 0.0464 & 0.45 & 0.227 & 0.30 & 0.0476 & 1.55 & $91.8 \%(56 / 61)$ & $90.8 \%(207 / 228)$ & $84.6 \%(165 / 195)$ \\
\hline Family history of Depression & 0.0585 & 0.26 & 0.542 & 0.09 & 0.0605 & 1.31 & $63.8 \%(44 / 69)$ & $73.5 \%(169 / 230)$ & $58.2 \%(113 / 194)$ \\
\hline Abnormal behavior & 0.0707 & 0.36 & 0.978 & 0.006 & 0.0859 & 1.43 & $88.4 \%(61 / 69)$ & $89.7 \%(217 / 242)$ & $82.6 \%(166 / 201)$ \\
\hline Sleep time during episodes $(\mathrm{h})$ & 0.0831 & 0.37 & 0.107 & 0.348 & - & - & $18.74 \pm 0.34(68)$ & $18.43 \pm 0.23(216)$ & $18.01 \pm 0.23(170)$ \\
\hline
\end{tabular}

We coded the minor allele additively and used linear regression for continuous variables and logistic regression for categorical variables: in model1, the minor allele genotype was fit as a function of the clinical variable; model2 was similar to model 1 except for including ethnicity, gender, and missing data proportion as three covariates. A Fisher's exact test of allelic counts was further performed for categorical variables. In these analyses, $592 \mathrm{KLS}$ cases were included (frequency \% | mean \pm SEM was computed). BB, allele G/G; AB, GGGAGCCA/G; AA, GGGAGCCA/GGGAGCCA; Minor Allele G (deletion) was the effect allele. 
Table 3. Association of clinical variables with genotype dose of TRANK1 deletion (rs71947865) after adjusting for medical history of birth difficulties, gender, referrer/race, and missing data proportion in 592 KLS cases (linear regression for continuous variables and logistic regression for categorical variables)

\begin{tabular}{|c|c|c|c|c|c|}
\hline Clinical variable & $P$ value & beta & $\mathrm{BB}(n=81)$ & $\mathrm{AB}(n=276)$ & $\mathrm{AA}(n=235)$ \\
\hline Severe cognitive symptoms during episodes & 0.011 & 0.99 & $97.1 \%(67 / 69)$ & $96.3 \%(233 / 242)$ & $90.4 \%(179 / 198)$ \\
\hline Age at KLS onset, y & 0.026 & 0.76 & $15.99 \pm 0.56(72)$ & $15.6 \pm 0.29(249)$ & $14.83 \pm 0.32(210)$ \\
\hline First episode duration, $d$ & 0.046 & 3.63 & $17.64 \pm 3.76(72)$ & $16.13 \pm 1.87(241)$ & $11.51 \pm 0.86(204)$ \\
\hline
\end{tabular}

Frequency $\% \mid$ mean \pm SEM was computed. *Minor Allele G (deletion) was the effect allele.

bipolar GWAS replication set (15), a caveat being that birth year data were not made available in this study (15) for all but one replication cohort where the birth year range was 1981-2005. Further, later reported TRANK1 ORs for bipolar disorder decreased in size from earlier studies in Brazil (40) and other countries (41-43) to the most recent metanalysis (15), even independently of the nonsignificant replication sample of this most recent metanalysis (15). This is illustrated in Dataset S3, where published TRANK1 association results are reported, with the caveat that the same samples are typically used cumulatively across studies.

In a subset of the full cohort (discovery and replication sets) when a valid birth year was available $(n=650)$, we performed TRANK1 SNP association by birth year stratified into three approximately equal-sized groups; 1987 or earlier $(n=220$, median year of birth: 1978), 1988-1994 $(n=202$, median year of birth: 1992), and 1995-2000s ( $n=228$, median year of birth: 1999). Using genetic principle component matched controls for each stratum mainly derived from the GERA cohort (birth year range 1928-1989), we observed a decrease in TRANK1 SNP rs71947865 association with time (Fig. $3 A$ and Dataset S6 $A$ ). Notably, while individuals born in 1987 or earlier had the strongest association TRANK1 association $\left(P=8.3 \times 10^{-4}\right.$; OR = $1.41)$, this effect decreased in KLS cases born 1988-1994 $(P=$ $\left.7.48 .3 \times 10^{-3} ; \mathrm{OR}=1.34\right)$ to finally disappear in the $1995-2000 \mathrm{~s}$ group $(P=0.31 ; \mathrm{OR}=1.11)$. In addition, within KLS cases, a
Fisher's test of allelic counts was performed, and birth year bin 1987 or earlier was compared to 1988-1994 and 1995-2000s in a $2 \times 2$ contingency table. We observed the highest association of TRANK1 SNP rs71947865 $(P=0.002)$ in year bin 1987 or earlier when compared to the minor allele count of TRANK1 SNP rs71947865 in the birth year bin 1995-2000s (Dataset S6B).

GWAS Analysis of Follow-up KLS Cases with and without Reports of Birth Difficulties. To replicate these findings, we collected and genotyped 171 new KLS cases collected between 2017 and 2019, collecting information on birth difficulties whenever possible. In the resulting sample set, birth difficulties data were available for 151 of the 171 cases. In the overall replication sample $(n=171)$, generally born more recently (median year of birth: 1998; range: 1960-2015), TRANK1 rs71947865 was not significantly associated with $\operatorname{KLS}(P=0.1, \mathrm{OR}=1.1)$ (Table 1 and Dataset $\mathrm{S} 2)$. Strikingly, however, when we stratified this sample into cases with versus without birth difficulties, then a significant TRANK1 rs71947865 association was seen only in the first group $(P=0.01$; $\mathrm{OR}=1.54$ in $n=59$ cases reporting birth difficulties; Dataset S7). In KLS cases with no birth difficulties, no TRANK1 rs71947865 effect was observed $(P=0.4$; OR $=1.12$ in $n=88$ cases). No birth information was available for $n=24$ patients. A combined TRANK1 SNP metaanalysis $\left(\mathrm{OR}=1.4 ; P=3.5 \times 10^{-8}\right)$ of both discovery and replication cohorts is presented in Table 1 .
A

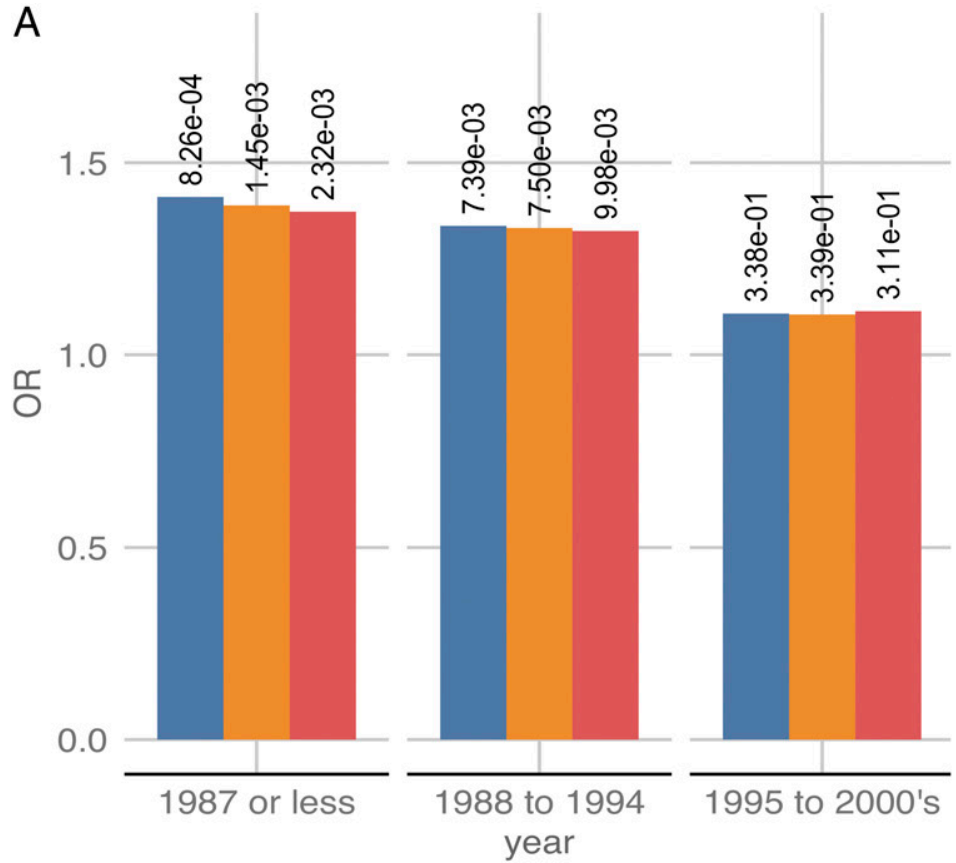

B

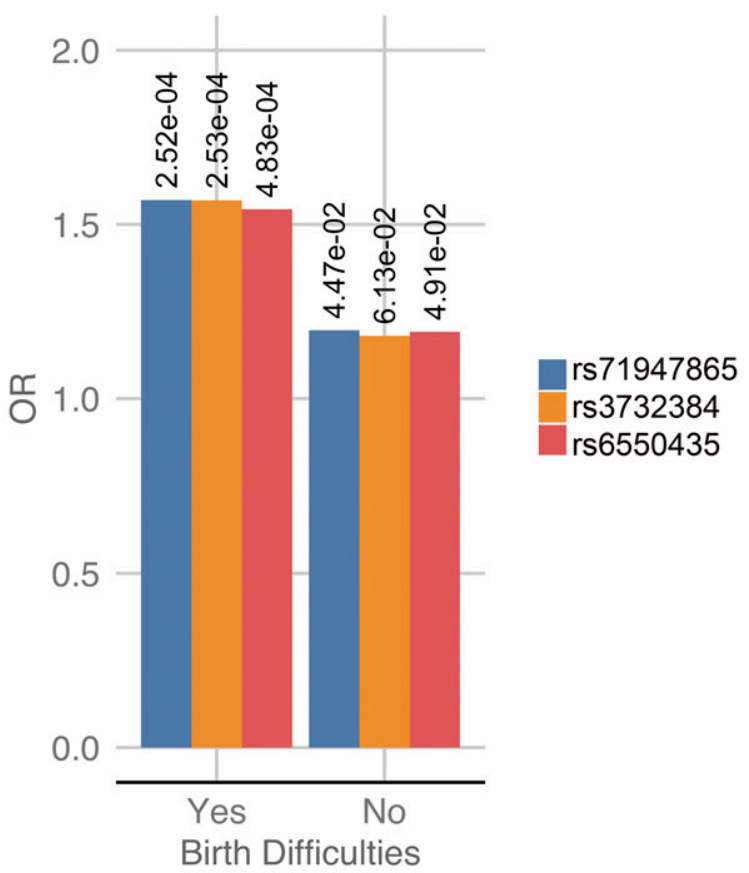

Fig. 3. (A) The TRANK1-region SNP associations in KLS cases stratified by birth years; the $y$ axis represents the odds ratio of the effect allele, while the $x$ axis is the birth year. $P$ value of the association is labeled. $(B)$ The TRANK1-region SNP associations in KLS cases stratified by birth difficulties; the $y$ axis represents the odds ratio of the effect allele, while the $x$ axis is the presence of birth difficulties. 
Our prior analyses of the discovery cohort had revealed significant association of TRANK1 variant rs71947865 with birth difficulties; we therefore determined the association of TRANK1 rs71947865 variants in KLS cases with birth difficulties when compared to controls individuals in both discovery and replication cohorts. We observed that TRANK1 rs71947865 variants were significantly associated with birth difficulties in KLS cases from both the discovery cohort $(P=0.002$; OR $=1.61$; see Dataset $\mathrm{S} 8 A)$ and the follow-up sample of KLS cases $(P=0.03$; $\mathrm{OR}=1.53)$. A combined analysis of KLS cases with and without birth difficulties from combining both the discovery and replication cohorts compared to genetic PCA matched controls also revealed significantly increased TRANK1 variant rs71947865 frequencies in KLS cases with birth difficulties (Fisher's test, $\mathrm{OR}=1.54 ; P=4.83 \times 10^{-4}$; Fig. $3 B$ and Dataset $\mathrm{S} 8 B$ ), while, in KLS cases without birth difficulties, the effect was weaker (Fisher's test, $\mathrm{OR}=1.20 ; P=0.04$ ).

Fine Mapping of the KLS Signal across Ethnicity. GWAS studies in schizophrenia and bipolar disorders have mapped the TRANK1 signal at SNP rs9834970 $\left(r^{2}=0.74\right.$ with KLS SNP rs71947865) in Caucasians (15, 42-44), while, when a mixed ethnic sample that included Asians and Caucasian was used, the top SNP is rs3732386 $(16,45-47)$, a signal entirely linked $\left(r^{2}=1\right)$ with the KLS-associated SNP rs71947865 (Dataset S3 and SI Appendix, Fig. S2 $A-C$ ).

Since our KLS sample is derived from multiple ethnicities, as an additional validation, we sought to determine TRANK1 loci association KLS cases of European/Caucasian origin only. In the overall European KLS metaanalysis, we observed that the $T R A N K 1$ region SNP rs71947865 $\left(\mathrm{OR}=1.28 ; P=1.9 \times 10^{-4}\right)$ was retained as the top association (Dataset S9 and SI Appendix, Fig. S2 $A-C$ ), with no evidence of heterogeneity. Our KLS European/ Caucasians-only analysis (discovery plus replication cohort) revealed that rs9834970 was among the top associations in the loci (SI Appendix, Fig. S2D and Dataset S9). The rs3732386 and rs71947865 were the top associations in the TRANK1 region and were dependent on the existence of associated birth difficulties (SI Appendix, Fig. S2). Further, on comparing the associations in the TRANK1 loci in discovery KLS versus combined schizophrenia and bipolar disorder GWAS, there is remarkable overlap of signals, with only the magnitude of ORs being 1.3× increased in KLS (SI Appendix, Fig. S2D).

Polygenic Risk Score of KLS. We found significant evidence that the KLS discovery cohort polygenic risk scores (PRSs) were indicative of increased genetic liability to KLS phenotype in a followup replication cohort $(n=171)$ under various $P$ value thresholds using PRSice2 (48). The most predictive $P$ value threshold was 0.5 (pseudo $R^{2} P=2 \times 10^{-22}$; Fig. 4) using 81,570 variants. $P$ value thresholds ranging from 0.05 to 0.4 were also indicative of increased genetic liability to $\operatorname{KLS}\left(P<1 \times 10^{-20}\right.$, mean pseudo $R^{2}=0.14$; see Dataset $\left.\mathrm{S} 10\right)$. Further, as an additional control, we also used only Caucasian KLS sample discovery metaanalysis ( $n=299)$ to build the PRS with validation only in Caucasian KLS replication sample $(n=160)$; the PRSs were indicative again of increased genetic liability to KLS (pseudo $R^{2}=0.094$ ), with the best score at $P=0.4$ threshold (SI Appendix, Fig. S3).

Considering the results above, we built PRSs using GWAS summary statistics from bipolar disorder (15), schizophrenia (49), circadian chronotype (50), sleep duration (51), and excessive daytime sleepiness (52), using previously published GWAS studies. These PRSs were used to score our KLS follow up cohort. Notably, shared KLS genetic liability (pseudo $R^{2}=0.06$ ) was found using bipolar disorder and schizophrenia GWAS summary statistics as the training set (SI Appendix, Fig. S3 and Dataset S10). LD score regression analysis estimated the total observed scale heritability $\mathrm{h} 2=0.09$ (intercept $=1.044$ ) and also revealed significant genetic correlation of KLS with bipolar disorder ( $\mathrm{rg}$ genetic correlation $=0.41$; $P<0.05)$ and depressive symptoms ( $\mathrm{rg}=0.32 ; P<0.05$; Dataset $\mathrm{S} 11)$.

Gene Pathway Analysis. Biological processes pathway enrichment analysis was next conducted using MAGMA (53). The MAGMA module gene-based association analysis was first performed in the combined discovery-replication sample summary statistics mapping SNPs to genes with a $1-\mathrm{kb}$ window upstream and downstream. This first step entails collapsing mean individual SNP statistics into a per gene-level statistic. Then a gene $P$ value is obtained by using a known approximation of the sampling distribution as detailed in MAGMA (53). The gene-based $P$ values converted to $\mathrm{z}$ scores are then used to conduct gene set enrichment analyses on gene sets derived from MsigDBv6.1 (5,346 curated gene sets). At 5\% FDR (false discovery rate), pathways "Reactome- RORA activates circadian expression" (54) and "Reactome-Circadian Repression of Expression By Rev ERBA" (55) were observed as significantly enriched in KLS genetic association analyses (Table 4). Notably, these two pathways shared identical gene members except for a single gene. Gene-based association tests within these enriched pathways are presented in Dataset S12. Both ROR Alpha (retinoic acid receptor-related alpha) and Rev ERBA are critical transcriptional regulators of periodic expression of the BMAl1 gene, directly regulating circadian pathways (56-59). Other circadian pathways of interest included "Reactome-Bmal1 Clock NPAS2 Activates Circadian Expression" but did not reach 5\% FDR threshold.

KLS Exome Sequencing Analysis. Targeted exome sequencing was performed in 32 individuals with KLS, derived from 16 multiplex families (Dataset $\mathrm{S} 13 A$ ). The variant frequencies and variant by variant analyses compared to 286 European controls from 1000 Genomes phase 3 were largely unremarkable. Damaging or probably damaging variants in each gene were collapsed, and mutational burden in KLS probands and/or shared by at least three families was identified and presented below.

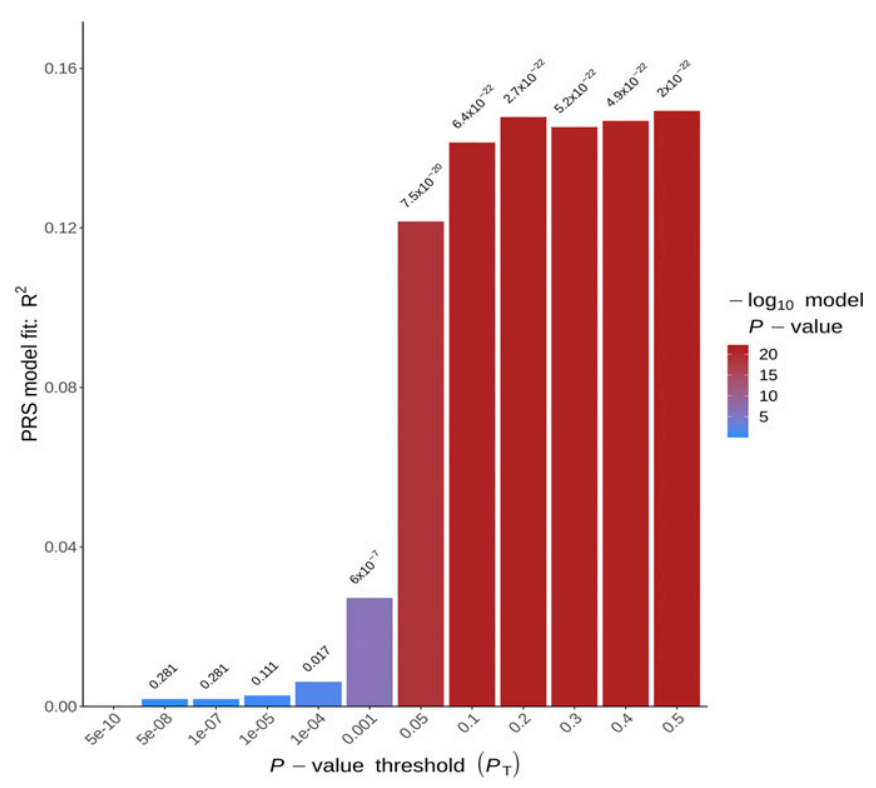

Fig. 4. Phenotypic variance explained by PRSs (pseudo $R^{2}$ ) built on the KLS discovery cohort and tested on the KLS replication cohort; the $y$ axis is the pseudo $R^{2}$, while the $x$ axis represents the $P$ value thresholds. The color gradient over each bar is representative of the $P$ value association between the PRS and the KLS trait in replication cohort. 
LMOD3 Gene Variants Are Not Associated with KLS. Following up on a recent study suggesting increased mutational load in the LMOD3 gene in familial KLS cases (60), we analyzed the LMOD3 burden in our exome sequencing cohort of KLS probands and sporadic cases $(n=17)$ compared to controls $(n=$ 286). We observed no significant differences in mutational burden in the LMOD3 gene between cases and controls $(P=0.13$; see Dataset S13B). Variant level analyses in KLS probands $(n=$ 17) revealed a previously reported single site rs35740823 (chr3:69171290; p.R83H) to be enriched in KLS cases (allelic $\mathrm{OR}=3.8 ; P=0.06$ ) as compared to controls (60) (Dataset $\mathrm{S} 13 B$ ). We therefore imputed this LMOD3 variant rs35740823 and analyzed for associations in 151 KLS cases and 478 controls derived from replication cohort and predominantly of European origin, but did not find any remarkable differences $(\mathrm{OR}=1.1$; $P=0.77$; Dataset $\mathrm{S} 13 B$ ).

Gene Mutational Burden Test in KLS. We also compared the overall frequency of modifier variants tagged using SnpEff (61) as moderate or high effects in all genes in all familial KLS cases versus 1000 Genome controls $(n=286)$ running gene-based mutational burden analyses (Dataset $\mathrm{S} 14 A$ ), matched for ethnicity and SNP principal components. Although significant effects were found, none were found in genes within the TRANK1 loci. However, we observed increased mutational burden in the rare alleles in genes NRXN2, GABRG2, CHORDC1, AATK, and CASP7 (FDR $P<1 \times 10^{-4}$ ) among probands $(n=17)$ relative to 1000 Genomes reference samples (Dataset $\mathrm{S} 14 A$ ). Genes with excessive rare alleles across each KLS family were next analyzed. Notably, GABRG2, CASP7, and ANKRD31, among other genes, were shared across at least three KLS families (Dataset S14B).

Further, pathway burden test from the list of 21 genes belonging to the circadian regulation pathway identified through the MAGMA analysis revealed significantly increased minor allele burden (Dataset S15A) in the whole KLS cohort $(n=32)$ compared to European origin controls. We also used pathway sets from msigDB6.1 to profile mutational burden in each pathway in KLS probands $(n=14)$. Notably, we found increased mutational burden in GO term GAMMA AMINOBUTYRIC $A C I D$ SIGNALING PATHWAY (FDR $P=0.007$ ) in KLS probands among other pathways that included many neuronal pathways (Dataset $\mathrm{S} 15 B$ ). This is in agreement with results of the gene burden test where we observed significantly increased burden in GABRG2 in KLS probands.

\section{Discussion}

Our study explores the interaction of genetic and environmental contributions to the pathophysiology of KLS. Results of GWAS revealed that TRANK1 gene variants were predisposing and associated with birth difficulties in KLS. PRS constructed on discovery GWAS analysis found strongly replicable PRS that overlapped with bipolar disorder and schizophrenia. Pathway analysis also revealed the involvement of circadian regulation genes.

This large study did not confirm findings made in other smaller genetic studies of KLS. For example, a 20 -y-old study had suggested an HLA-DQ2 association (12) in KLS, but this was not confirmed either in other studies $(3,8,13)$ or in our current analyses. A recent study (60) focused on a large Saudi pedigree KLS-affected family where an rs111848977 p.E142D mutation in LMOD3 was observed to cosegregate. This study also reported an increased burden of damaging mutations, including p.R23H in LMOD3 in KLS, a gene involved in nemaline myopathy 10 (62). In our sample of 32 familial cases, we could not find any overall increase in the frequency of LMOD3 mutations, and, although p. R83H was nominally increased in familial cases, we did not find this association in a larger cohort of 151 KLS cases (Dataset S13B).

Although the phenomenology of KLS is quite different from that of bipolar disorder or schizophrenia, these syndromes have some clinical similarities. Most notably, in both KLS and bipolar disorder, patients are asymptomatic between episodes. Similarly, exacerbations are usually episodic in some schizophrenia. In KLS, however, onset/offset of episodes is rapid (typically within 1 d), while it is generally slower in most bipolar patients, except for "rapid cyclers" (63). Further, although not a dominant feature, depression, anxiety, or hallucinations/delusions can occur during KLS episodes $(3,4,6,64)$, and, at the end of episodes, some patients may have brief ( $1 \mathrm{~d}$ to $2 \mathrm{~d}$ ) period of elation, insomnia, and logorrhea which can resemble a manic miniepisode.

Most interestingly, hypersomnia is a common feature of depressive episodes in bipolar type 1 disorder (65-67), unlike in other depressions, where patients most often exhibit insomnia. Like KLS, bipolardisorder is also associated with cognitive deficiencies, although these are milder $(68,69)$ as compared to KLS patients. This, together with the fact that lithium is the only treatment reported to have an effect in preventing KLS episodes (7), suggests possible shared pathophysiology with bipolar disorder. Pathophysiological overlap among KLS, bipolar disorder, and schizophrenia is also in line with genetic studies in the UK Biobank cohort that have shown that the genetic architecture of long self-reported sleep duration overlaps with that of bipolar disorder, in contrast with the genetic architecture of insomnia that shares a similarity with anxiety and restless leg syndrome (70). Most strikingly, strong correlations between PRS scores of long sleep duration and bipolar disorder type 1 in the UK Biobank sample were found (71). This suggests that hypersomnia, periodic or not, could share some commonality with bipolar disorder and schizophrenia.

One possible hypothesis is that KLS, like bipolar disorder and other psychosis, is characterized by periodic instability of specific brain networks involving, in the case of KLS, reduced metabolism in cortical and subcortical areas, as reported by imaging studies (72). In this syndrome, cognitive and derealization symptoms may correlate with decreased activity in cortical areas, whereas decreased activity in thalamus and hypothalamus may be involved in the functional hypersomnia observed in disorder (72-74). Involvement of circadian or behavioral rhythmicity genes may be essential for maintaining hypersomnia episodes once an episode is initiated, for example, if subjects are suddenly spending much time in bed, unexposed to light, a behavior which could result in difficulties

Table 4. A gene set enrichment analysis was carried out on the combined discovery and follow-up cohort of KLS cases using the MAGMA module

\begin{tabular}{|c|c|c|c|c|}
\hline Gene set name & Ngenes & Beta & $P$ value & FDR $P$ value \\
\hline Reactome_Rora_Activates_Circadian_Expression & 22 & 1.03 & $1.29 \times 10^{-7}$ & 0.00069 \\
\hline Reactome_Circadian_Repression_Of_Expression_By_Rev_Erba & 21 & 0.97 & $2.17 \times 10^{-6}$ & 0.00579 \\
\hline Go_Regulation_Of_Tumor_Necrosis_Factor_Superfamily_Cytokine_Production & 88 & 0.29 & 0.00050 & 0.61043 \\
\hline Reactome_Bmal1_Clock_Npas2_Activates_Circadian_Expression & 33 & 0.51 & 0.00057 & 0.61043 \\
\hline
\end{tabular}

A total of 5,346 sets including curated gene sets, KEGG pathways, Reactome pathways, and GO biological processes derived from msigdb6. 1 were used (5\% FDR $P$ value was computed). Beta is the direction of association of the pathway/gene set with the KLS case control status. Ngenes is the number of genes. 
entraining in genetically susceptible individuals (75). This hypothesis could also explain why patients with KLS can exhibit sleep onset REM periods during Multiple Sleep Latency Test (MSLT; the circadian clock strongly drives occurrence of REM sleep) (76, 77), a feature also associated with shift work (78). Other investigators have found abnormal circadian entrainment during KLS episodes using actigraphy (79). A role for circadian abnormalities has also been suggested in bipolar disorder type $1(67,80,81)$. Subjects with bipolar type 1 also reported increased eveningness tendencies (71), although this was not found in patients with KLS (3).

The most striking finding of this study is the observation of a strong genome-wide significant association of KLS with TRANK1region variants (OR of $\sim 1.50$ ) that are strongly associated with bipolar disorder (OR of 1.12) $(43,82,83$ ) and schizophrenia (OR of 1.08) (46, 84-86), but not mood instability (87). Although it remains possible this effect could be due to a rarer variant linked with this haplotype in KLS, this is more likely mediated by a similar effect of these common variants across these diseases. Of notable importance is the fact the TRANK1 effect size in this study is four to five orders of magnitude higher (OR of $~ 1.5)$ than in studies in these other pathologies (OR of $\sim 1.1$ ), explaining detection of association in a small sample of less than 1,000 KLS patients. This association with KLS is thus unlikely to be explained by contamination of our current KLS cohort with bipolar cases, although the opposite, contamination of bipolar or schizophrenia samples with KLS patients, could explain weaker associations in these other pathologies.

The reason for the weaker than expected TRANK1 replication in the KLS replication cohort became evident after stratifying our discovery sample by birth decade, finding that the TRANK1 association was highest in subjects born before the 1980s and decreased thereafter. Further, KLS subjects with TRANK1 polymorphism have reported increased birth difficulties (summarized in Dataset S5). We hypothesize that improved perinatal outcomes that have occurred in the past 50 y may explain this tendency (34-39) (https://www.cdc.gov/nchs/data/nvsr/nvsr56/nvsr56_10.pdf; https:// mchb.hrsa.gov/sites/default/files/mchb/Data/Chartbooks/child-health2014.pdf). The TRANK1 birth difficulties relationship was also confirmed in the independent replication sample, where we found that only the subset of KLS patient reporting birth difficulties had a significant TRANK1 association. Overall, patients who reported birth difficulties had the highest association (Fisher's test, OR = $\left.1.57 ; P=2.5 \times 10^{-4}\right)$ in both the discovery and replication cohorts (Dataset S9). TRANK1 association also increases disease severity during episodes independently of birth difficulty effects (Table 3 ).

Interestingly, although the association with TRANK1 was the highest genome-significant finding in bipolar disorder in a recent metanalysis of 32 cohorts from 14 countries in Europe, North America, and Australia, totaling 20,352 cases and 31,358 controls of European descent (rs9834970; OR $=1.10, P=5.5 \times 10^{-14}$ ) (15), as in our current KLS sample, replication in follow-up bipolar disorder samples totaling 9,412 cases and 137,760 controls of European ancestry did not replicate $(\mathrm{OR}=1.02, P=0.15)$. This finding deviated more than expected by chance $(P=0.01$, although not significant if Bonferroni corrected for all loci detected in the study). We hypothesize that a similar time-dependent TRANK1 birth difficulty interaction may play a role in bipolar disorder and schizophrenia. Indeed, these disorders are known to be strongly associated with early-life complications $(17-28,88,89)$, and data in the UK Biobank cohort indicate a dose-dependent increased prevalence of major depressive disorders and bipolar disorders in association with reported low birth weight (90), even when controlled for a history of the physical disorder known to be associated with low birth weight.

Two possibilities, which are not necessarily mutually exclusive, could explain these data. First, TRANK1-region genes could affect placental physiology, modulating the risk of birth difficulties and long-term fetal damage that could manifest as KLS or other disorders. Ursini et al. (31) found strong significant genetic interaction between the PRS of schizophrenia and the occurrence of early-life complications, in multiple case-control samples. Further, MLH1, LRRFIP2, and GOLGA4 associated with these overall genetic components were highly expressed in the placenta and modulated by pathological placental processes such as preeclampsia and intrauterine growth restriction, but not in other disease processes (31). Problematically, however, recent papers did not observe that the KLS-associated SNPs identified in this study had substantial effects on placental expression of these genes $(31,32,91,92)$. Further, TRANK1-region polymorphisms are not known maternal or fetal genetic factors associated with low birth weight (93-95), prematurity (96), or birth complications $(91,97)$ in large samples, and were not associated with birth difficulties in a small sample of 198 controls ascertained similarly to our KLS subjects for birth difficulties. Finally, this hypothesis would not explain the time dependency/cohort effect of the TRANK1 association we observed with KLS, which is likely due to improved or changes in perinatal care with time.

Another possible explanation, which we favor as it better fits our results and the lack of effects of TRANK1 on birth difficulties in previously published studies, is that newborns carrying $T R A N K 1$-region polymorphisms are more susceptible to injury in the presence of birth difficulties (notably hypoxia) in a way that produces behavioral instability such as KLS or other psychiatric conditions such as bipolar disorder in adulthood. This would be a true gene $\times$ environment interaction, although our study lacked sufficient control to formally test this hypothesis statistically. The mechanism underlying this phenomenon, which could have evolutionary advantages in times of stress (98), remains to be investigated. Interestingly, rs9834970, an SNP linked with the TRANK1region susceptibility haplotype, is associated with decreased expression of TRANK1 in induced pluripotent stem cells (iPSCs) and neural progenitor cells (99); such an effect has not been reported in the adult brain, but, as discussed, TRANK1-associated polymorphism reduces expression in various dividing tissues such as blood, esophageal mucosa, and B cells. The iPSC/neuronal precursor cell effect was rescued by valproic acid but not lithium, and the TRANK1 gene knockdown was found to modulate the expression of many genes, suggesting developmental effects (99).

Because the susceptibility haplotype modulates expression of many other genes in a tissue-specific manner, however, we believe the study of other genes in this region across tissues and development with the susceptibility haplotype will be necessary before concluding that TRANK1 is the gene behind this genetic association, which is a limitation of our analyses. Additional studies aiming at studying more precisely which types of birth difficulties are associated with TRANK1 in these disorders are also needed. Our results offer clues on the developmental window in which the TRANK1-susceptibility haplotype could affect risk for KLS and mental disorders in adulthood and mandate a more careful analysis of what and how the reported birth difficulties interact with specific TRANK1 genetic variants.

Our data support a genetic predisposition in the presence of $T R A N K 1$ polymorphisms and link KLS, circadian regulation, and bipolar disorder. PRSs derived from discovery KLS samples are strongly predictive of the KLS phenotype in the replication sample. Further, PRSs support a genetic overlap with bipolar disorder and schizophrenia, with a caveat being that these associations in various $P$ thresholds were unadjusted for multiple comparisons and likely are underpowered, since only the replication follow-up KLS sample was used. Strikingly, the temporal effect of TRANK1 polymorphism was observed in KLS cases in relation to the year of birth. In the presence of birth difficulties, the TRANK1 polymorphism may predispose to pathophysiological consequences such as KLS, bipolar disorder, and schizophrenia. 


\section{Materials and Methods}

Inclusion Criteria for KLS and Origin of Cases. Patients with KLS were included if they met International Classification of Sleep Disorders (ICSD3) criteria for the disorder, including the presence of recurrent episodes $(\geq 2)$ of severe hypersomnia ( $>18 / 24 \mathrm{~h}$ ) lasting days to weeks, associated with cognitive disturbances (confusion, amnesia, and derealization) and behavioral abnormalities (major apathy, megaphagia, and hypersexuality). These episodes had to be interspersed with long periods of normal sleep, cognition, behavior, and mood. A sleep-trained physician diagnosed all patients. They were also asked to answer a detailed questionnaire, or a systematic interview as described $(3,6)$. Informed consent, in accordance with governing institutions, was obtained from all subjects. The research protocol was approved by Institutiona Review Board (IRB) panels on Medical Human Subjects at Stanford University, and by respective IRB panels in each country providing samples for the study.

KLS Cases. The discovery sample of 673 KLS cases includes 261 cases recruited in the United States, with the help of the Kleine-Levin Syndrome Foundation (KLSF). US patients were diagnosed by an outside physician or referred by the KLSF (N.F.), and the diagnosis was confirmed at Stanford, where the questionnaire and blood sample were collected. Other major recruitment sites include Pitié-Salpêtrière, Paris, France (I.A., $n=140$ ); University of Peking, Beijing, People's Republic of China, (F.H., $n=62$ ); Taiwan University Taipei, Republic of China, Taiwan (Y.-S.H., $n=58$ ); Guy de Chauliac Hospital, Montpellier, France (Y.D., $n=35$ ); Safra Children's Hospital, Sheba Medica Center, Tel Aviv University, Tel Aviv, Israel (Y.S., $n=34$ ); Hepata Clinic, Germany (G.M., $n=24$ ); Uppsala University, Sweden (A.M.L., $n=15$ ); Hospital Robert Debre, Paris, France (M.L., $n=7$ ); Tokyo Metropolitan Institute of Medical Science Tokyo, Japan (M.H., $n=7$ ); University of Bologna, Italy (F.P. and G.P., $n=6$ ); Maynei Hayeshua Medical Center, Bnei Barak, and The Sackler Faculty of Medicine, Tel-Aviv University (N.G., $n=6$ ); Akita University, Akita, Japan (T.K., $n=2$ ); St. Vincent's Hospital, The Catholic University of Korea (S.C.H., $n=2$ ); 1st Faculty of Medicine and General Teaching Hospital, Prague, Czech Republic (S.N., $n=2$ ); Bambino Gesù Children Hos pital, Rome, Italy (Marina Macchialio, $n=2$ ); and other centers who contributed single sample $(n=10)$.

The replication cohort included 171 additional KLS cases. These included cases from Pitié-Salpêtrière, Paris, France (I.A., S.L.-S., and P.D., $n=66$ ) Uppsala University, Sweden (A.M.L., $n=26)$; KLSF $(n=23)$; University of Bologna, Italy (F.P. and G.P., $n=13$ ); London Bridge Hospital, London, United Kingdom (G.D.L., $n=11$ ); Guy de Chauliac Hospital, Montpellier, France (Y.D. $n=9$ ); Emory University, Atlanta (D.R., $n=6$ ); Hepata Clinic, Germany (G.M. $n=5)$; Stanford University, (E.J.-M.M., $n=5)$; Tokyo Metropolitan Institute of Medical Science, Tokyo, Japan (M.H., $n=4)$; and other centers $(n=3)$.

GWAS Patients and Matched Controls. Because the samples were collected over a long time frame (2003-2019), genotyping platforms evolved, and hence each platform required its cohort of cases and controls (Dataset S1). As a result, the discovery sample ( $n=673,2003-2017)$ was subdivided into seven subcohorts of cases and controls based on genotyping platform/ethnicity differences. Cases and ethnically matched controls $(n=15,339)$ were genotyped at University of California, San Francisco genomics core with Affymetrix Axiom Genome-Wide LAT, EUR, CHB, Axiom 55066 arrays, and Axiom world arrays, while multiethnic Axiom PMRA arrays were genotyped at Akesogen Inc. These include three European ancestry cohorts, an Asian cohort, a Latino cohort, and Ashkenazi and non-Ashkenazi Jewish cohorts. The replication sample ( $n=171$, collected between the years 2017 and 2019), subcohort 8, was treated as a single sample and genotyped solely on Axiom PMRA arrays. DNA was extracted using Qiagen DNeasy kit, and samples were stored at $-20^{\circ} \mathrm{C}$ until genotyping; a minimum of $\sim 200 \mathrm{ng}$ was used for genotyping.

Genotype Analysis and Imputation. Raw CEL files were imported into Affymetrix Genotyping console, and Affymetrix best practices workflow was followed. Genotype calls were exported as plink ped/map files (100). For CEL files generated from Axiom world arrays, primarily AffyPipe was used to call the genotypes and exported as plink ped/map files (101). Stringent quality control (QC) was applied, variants and individuals with $>0.05$ missingness were excluded, related individuals with identity by descent pihat $>0.2$ were excluded, and markers with Hardy-Weinberg equilibrium $P<1 \mathrm{e}^{-6}$ in controls were excluded. HapMap and 1000 Genomes phase 3 reference population genotypes (102) were used to guide ethnicity matching in our sample subcohorts. Genetic Prinicipal Components (PCs) were extracted using plink. PC plots were visually inspected for unexpected deviations and outliers, after which Euclidean distance was used to accurately and automatically match cases and controls. Post QC, genotypes were phased one chromosome at a time with shapeit $(103,104)$ using the build37 coordinate system of the human genome. Phased haplotypes were then subject to imputation to 1000 Genomes phase 1 in 1-mb chunks for each chromosome, using impute2 (105).

Association Testing and Variant Prioritization. Frequentist association tests were then performed on the imputed genotypes by including genetic principal components (PCA) and missing data proportion using snptest (106). Metaanalysis was subsequently performed using cohort-specific $\beta$ estimates using a fixed effects model (106). The genome-wide significance threshold was set at $P<5 \mathrm{e}-8$ based on Bonferroni adjustment. Gene-based association tests, including pathway analysis, were performed with MAGMA modules (107). Manhattan plots were constructed with R package qqman (108). Additional eQTL annotation was carried out using SNIPA, webserver (109), and GTEx v8 (30). eCAVIAR was used to perform eQTL colocalization (110). LD hub was used to calculate LD score regression and find shared SNP heritability across phenotypes (111). PRSs were computed using PRSice2 (48), briefly imputed genotypes in BGEN format from the replication dataset were quality controlled (minor allele frequency $>0.05$ and impute info score $\geq 0.9$ ) and clumped for LD ( $250-\mathrm{kb}$ window; $r 2=0.1$ ) and used as a target dataset along with the first five genetic PCAs. The number of common variants remaining after clumping from the base and target dataset were 104626. Apart from using summary statistics from the KLS discovery, we also used summary statistics from various other phenotypes. PRSs at various $P$ value thresholds $\left(5 \times 10^{-10}, 5 \times 10^{-8}, 1 \times 10^{-7}\right.$, $1 \times 10^{-5}, 0.0001,0.001,0.05,0.1,0.2,0.3,0.4,0.5$, and 1) were derived and validated in KLS replication cohort $(n=171)$.

Exome Sequencing Sample and Matched Controls. Agilent Sure Select V4 system workflow was used to capture regions of interest; the resulting fragments were subject to Illumina paired-end sequencing run with an average depth of $130 \times$ and average read length of 100 in the target regions for the KLS family DNA samples. The KLS family DNA consisted of 32 individuals derived from 16 multiplex families (Dataset S13A). On the other hand, for controls, exome FASTQ files from 286 individuals part of 1000 Genomes (predominantly CEU, TSI, GBR, IBS, and FIN) were used. The FASTQ files were aligned to UCSC hg19 reference FASTA using BWA (112) and read groups inserted for downstream variant quality score recalibration. GATK best practices workflow (https://gatk.broadinstitute.org/hc/en-us/sections/ 360007226651) was implemented, and joint genotyping was performed on both cases and controls simultaneously. Raw variant call sets were subject to variant quality score recalibration, and variants passing the filters were then imported into plinkseq (https://www.atgu.mgh.harvard.edu/resources/software/) and used in downstream association analysis. Annovar and SnpEff were used to annotate variants $(61,113)$, following which moderate and high modifier variants were collapsed, and gene-based burden tests in various subcohorts were carried out using plinkseq.

A gene burden test was carried out within each family, probands only and all 32 individuals. Each analysis used the 1000 Genomes European dataset matched by principle components as a control cohort. The burden analyses were focused only in rare variants (minor allele counts 2 to 20) and restricted to predicted to be moderate or high modifiers as tagged by Annovar and SnpEff. Further, genes that were part of the rhythmic behavior pathway were collapsed, and SKAT-based (sequence kernel association test) gene burden tests were performed (114) with resulting $P$ values adjusted using a $5 \%$ FDR.

Taqman Genotyping. To validate the genotype calls from SNP genotyping arrays, we conducted Taqman assays for TRANK1 SNPs rs71947865 and rs6550435 on a random subset of KLS cases and controls typed across multiple platforms $(n=122)$. Allele concordance for rs71947865 was $100 \%$, while, for rs6550435, it was $97 \%$ in 122 subjects.

Clinical Associations. Demographic and various clinical features within the KLS cohort $(n=592)$ were analyzed for associations with TRANK1 rs71947865 variant (deletion). Three kinds of analyses were performed: In model 1, the minor allele genotype (additively coded) was fit as a function of the clinical variable; model 2 was similar to model 1 except for including ethnicity, gender, and missing data proportion as three covariates. A Fisher's exact test of allelic counts was further performed for categorical variables.

Birth Year Associations. Two analysis strategies were pursued. First, when birth year was available for KLS cases $(n=650)$, we stratified the KLS cases into three approximately equal-sized groups: 1987 or before ( $n=220$, median year of birth: 1978), 1988-1994 ( $n=202$, median year of birth: 1992), and 1995-2000s ( $n=228$, median year of birth: 1999). Within each group, we matched control samples based on genetic principle components. Fisher's exact and $\chi^{2}$ tests on TRANK1 rs71947865 allelic counts were performed to 
find significant difference in allele frequencies. A Fisher's test of TRANK1 SNPs allelic counts was performed within KLS cases stratified by the three birth year bins; the minor allele counts in birth year bin 1987 or earlier was compared to minor allele counts 1988-1994 and 1995-2000s in $2 \times 2$ contingency table.

Data Availability. All the analyses presented in the manuscript is from data in Datasets $\mathrm{S} 1$ to $\mathrm{S} 15$ which are available. Complete summary statistics of the genetic associations are available at https://doi.org/10.6084/m9.figshare. 14128475.v2.To protect the privacy of the study participants, the individual genetic data generated and analyzed during this study are available from the corresponding authors on reasonable request.

1. D. W. Kleine, Periodische Schlafsucht. Eur. Neurol. 57, 305-320 (1925)

2. M. Levin, Periodic somnolence and morbid hunger: A new syndrome. Brain $\mathbf{5 9}$ 494-504 (1936)

3. I. Arnulf et al., Kleine-Levin syndrome: A systematic study of 108 patients. Ann Neurol. 63, 482-493 (2008).

4. I. Arnulf, T. J. Rico, E. Mignot, Diagnosis, disease course, and management of patients with Kleine-Levin syndrome. Lancet Neurol. 11, 918-928 (2012).

5. M. Billiard, I. Jaussent, Y. Dauvilliers, A. Besset, Recurrent hypersomnia: A review of 339 cases. Sleep Med. Rev. 15, 247-257 (2011).

6. I. Arnulf, J. M. Zeitzer, J. File, N. Farber, E. Mignot, Kleine-Levin syndrome: A systematic review of 186 cases in the literature. Brain 128, 2763-2776 (2005).

7. S. Leu-Semenescu et al., Lithium therapy in Kleine-Levin syndrome: An open-label, controlled study in 130 patients. Neurology 85, 1655-1662 (2015).

8. Y. S. Huang et al., Relationship between Kleine-Levin syndrome and upper respiratory infection in Taiwan. Sleep (Basel) 35, 123-129 (2012).

9. Q. T. Nguyen et al., Familial Kleine-Levin syndrome: A specific entity? Sleep (Basel) 39, 1535-1542 (2016)

10. R. Peraita-Adrados, J. L. Vicario, M. Tafti, M. García de León, M. Billiard, Monozygotic twins affected with Kleine-Levin syndrome. Sleep (Basel) 35, 595-596 (2012).

11. O. Habra, R. Heinzer, J. Haba-Rubio, A. O. Rossetti, Prevalence and mimics of KleineLevin syndrome: A survey in French-speaking Switzerland. J. Clin. Sleep Med. 12, 1083-1087 (2016).

12. Y. Dauvilliers et al., Kleine-Levin syndrome: An autoimmune hypothesis based on clinical and genetic analyses. Neurology 59, 1739-1745 (2002).

13. S. Lavault et al., Kleine-Levin syndrome in 120 patients: Differential diagnosis and long episodes. Ann. Neurol. 77, 529-540 (2015).

14. M. Lam et al.; Schizophrenia Working Group of the Psychiatric Genomics Consor tium; Indonesia Schizophrenia Consortium; Genetic REsearch on schizophreniA neTwork-China and the Netherlands (GREAT-CN), Comparative genetic architecture of schizophrenia in East Asian and European populations. Nat. Genet. 51, 1670-1678 (2019).

15. E. A. Stahl et al.; eQTLGen Consortium; BIOS Consortium; Bipolar Disorder Working Group of the Psychiatric Genomics Consortium, Genome-wide association study identifies 30 loci associated with bipolar disorder. Nat. Genet. 51, 793-803 (2019).

16. M. Ikeda et al., Genome-wide association study detected novel susceptibility genes for schizophrenia and shared trans-populations/diseases genetic effect. Schizophr. Bull. 45, 824-834 (2019).

17. S. A. Stilo, R. M. Murray, Non-genetic factors in schizophrenia. Curr. Psychiatry Rep. 21, 100 (2019).

18. J. Söderlund, S. Wicks, L. Jörgensen, C. Dalman, Comparing cohort incidence of schizophrenia with that of bipolar disorder and affective psychosis in individuals born in Stockholm County 1955-1967. Psychol. Med. 45, 3433-3439 (2015).

19. R. Chudal et al., Perinatal factors and the risk of bipolar disorder in Finland. J. Affect Disord. 155, 75-80 (2014).

20. U. K. Haukvik et al., Pre- and perinatal hypoxia associated with hippocampus/ amygdala volume in bipolar disorder. Psychol. Med. 44, 975-985 (2014).

21. C. Nosarti et al., Preterm birth and psychiatric disorders in young adult life. Arch Gen. Psychiatry 69, E1-E8 (2012).

22. J. McGrath, J. Scott, Urban birth and risk of schizophrenia: A worrying example of epidemiology where the data are stronger than the hypotheses. Epidemiol. Psichiatr. Soc. 15, 243-246 (2006).

23. M. Cannon, P. B. Jones, R. M. Murray, Obstetric complications and schizophrenia: Historical and meta-analytic review. Am. J. Psychiatry 159, 1080-1092 (2002)

24. H. Kunugi, S. Nanko, R. M. Murray, Obstetric complications and schizophrenia: Prenatal underdevelopment and subsequent neurodevelopmental impairment. $B r$. J. Psychiatry Suppl. 40, s25-s29 (2001).

25. D. K. Kinney, D. A. Yurgelun-Todd, M. Tohen, S. Tramer, Pre- and perinatal complications and risk for bipolar disorder: A retrospective study. J. Affect. Disord. 50 117-124 (1998)

26. J. R. Geddes, S. M. Lawrie, Obstetric complications and schizophrenia: A metaanalysis. Br. J. Psychiatry 167, 786-793 (1995).

27. T. F. McNeil, Perinatal risk factors and schizophrenia: Selective review and meth odological concerns. Epidemiol. Rev. 17, 107-112 (1995).

28. S. N. Brixey, B. J. Gallagher 3rd, J. A. McFalls Jr., L. F. Parmelee, Gestational and neonatal factors in the etiology of schizophrenia. J. Clin. Psychol. 49, 447-456 (1993).

29. H. Shimizu, A. Hirose, T. Tatsuno, M. Nakamura, J. Katsube, Pharmacological properties of SM-3997: A new anxioselective anxiolytic candidate. Jpn. J. Pharmacol. 45, 493-500 (1987).

30. GTEx Consortium, The genotype-tissue expression (GTEx) project. Nat. Genet. 45, 580-585 (2013).
ACKNOWLEDGMENTS. We thank countless staff members, clinicians, patients, and patient advocates who are not listed here and have provided material and logistic support over decades for these 15-y-long studies. The study was primarily funded by the KLSF (2003-2020) and by NIH Grant 1R01MH080957 (2007-2013) to E.J.-M.M. The French Kleine-Levin Syndrome research program is financed by a grant to I.A. (Grant PHRC 070138) from the French Ministry of Health. H.M.O. has been supported by the Academy of Finland (Grant 309643), Yrjö Jahnsson Foundation, and Oskar Öflund Foundation. Computing for this project was performed on the Stanford Genomics cluster supported by NIH Grant 1S10OD023452-01. We thank the Stanford Research Computing Center for providing computational resources and support that contributed to these research results.

31. G. Ursini et al., Convergence of placenta biology and genetic risk for schizophrenia. Nat. Med. 24, 792-801 (2018)

32. S. Peng et al., Expression quantitative trait loci (eQTLs) in human placentas suggest developmental origins of complex diseases. Hum. Mol. Genet. 26, 3432-3441 (2017).

33. T. J. Hoffmann et al., Next generation genome-wide association tool: Design and coverage of a high-throughput European-optimized SNP array. Genomics 98, 79-89 (2011).

34. A. Sander, R. Wauer, From single-case analysis of neonatal deaths toward a further reduction of the neonatal mortality rate. J. Perinat. Med. 47, 125-133 (2018).

35. M. K. Sugai, S. Gilmour, E. Ota, K. Shibuya, Trends in perinatal mortality and its risk factors in Japan: Analysis of vital registration data, 1979-2010. Sci. Rep. 7, 46681 (2017).

36. E. B. Schneider, Fetal health stagnation: Have health conditions in utero improved in the United States and Western and Northern Europe over the past 150 years? Soc. Sci. Med. 179, 18-26 (2017).

37. E. Verstraete et al., Healthcare-associated bloodstream infections in a neonatal in tensive care unit over a 20-year period (1992-2011): Trends in incidence, pathogens, and mortality. Infect. Control Hosp. Epidemiol. 35, 511-518 (2014).

38. S. V. Glinianaia et al., Temporal changes in key maternal and fetal factors affecting birth outcomes: A 32-year population-based study in an industrial city. BMC Pregnancy Childbirth 8, 39 (2008)

39. D. Rush, J. M. Alvir, D. A. Kenny, S. S. Johnson, D. G. Horvitz, The National WIC evaluation: Evaluation of the special supplemental food program for women, in fants, and children. III. Historical study of pregnancy outcomes. Am. J. Clin. Nutr. $\mathbf{4 8}$, 412-428 (1988).

40. R. Secolin et al., Family-based association study for bipolar affective disorder. Psychiatr. Genet. 20, 126-129 (2010).

41. A. W. Charney et al., Evidence for genetic heterogeneity between clinical subtypes of bipolar disorder. Transl. Psychiatry 7, e993 (2017).

42. L. Hou et al., Genome-wide association study of 40,000 individuals identifies two novel loci associated with bipolar disorder. Hum. Mol. Genet. 25, 3383-3394 (2016).

43. T. W. Mühleisen et al., Genome-wide association study reveals two new risk loci for bipolar disorder. Nat. Commun. 5, 3339 (2014)

44. D. M. Ruderfer et al.; Schizophrenia Working Group of the Psychiatric Genomics Consortium; Bipolar Disorder Working Group of the Psychiatric Genomics Consortium; Cross-Disorder Working Group of the Psychiatric Genomics Consortium, Polygenic dissection of diagnosis and clinical dimensions of bipolar disorder and schizophrenia. Mol. Psychiatry 19, 1017-1024 (2014)

45. Z. Li et al., Genome-wide association analysis identifies 30 new susceptibility loci for schizophrenia. Nat Genet. 49, 1576-1583 (2017).

46. Schizophrenia Working Group of the Psychiatric Genomics Consortium, Biological insights from 108 schizophrenia-associated genetic loci. Nature 511, 421-427 (2014).

47. D. T. Chen et al.; BiGS, Genome-wide association study meta-analysis of European and Asian-ancestry samples identifies three novel loci associated with bipolar disorder. Mol. Psychiatry 18, 195-205 (2013)

48. S. W. Choi, P. F. O'Reilly, PRSice-2: Polygenic Risk Score software for biobank-scale data. Gigascience 8, giz082 (2019).

49. D. M. Ruderfer et al., Genomic dissection of bipolar disorder and schizophrenia, including 28 subphenotypes. Cell 173, 1705-1715.e16 (2018).

50. S. E. Jones et al., Genome-wide association analyses of chronotype in 697,828 individuals provides insights into circadian rhythms. Nat. Commun. 10, 343 (2019)

51. H. S. Dashti et al., Genome-wide association study identifies genetic loci for selfreported habitual sleep duration supported by accelerometer-derived estimates. Nat. Commun. 10, 1100 (2019).

52. H. Wang et al., Genome-wide association analysis of self-reported daytime sleepiness identifies 42 loci that suggest biological subtypes. Nat. Commun. 10, 3503 (2019).

53. C. A. de Leeuw, J. M. Mooij, T. Heskes, D. Posthuma, MAGMA: Generalized gene-set analysis of GWAS data. PLoS Comput. Biol. 11, e1004219 (2015).

54. V. Giguère et al., Isoform-specific amino-terminal domains dictate DNA-binding properties of ROR alpha, a novel family of orphan hormone nuclear receptors. Genes Dev. 8, 538-553 (1994).

55. P. Griffin et al., Circadian clock protein Rev-erb $\alpha$ regulates neuroinflammation. Proc. Natl. Acad. Sci. U.S.A. 116, 5102-5107 (2019)

56. Y. Zhang et al., GENE REGULATION. Discrete functions of nuclear receptor Rev-erbo couple metabolism to the clock. Science 348, 1488-1492 (2015).

57. L. A. Solt, D. J. Kojetin, T. P. Burris, The REV-ERBs and RORs: Molecular links between circadian rhythms and lipid homeostasis. Future Med. Chem. 3, 623-638 (2011). 
58. F. Guillaumond, H. Dardente, V. Giguère, N. Cermakian, Differential control of Bmal1 circadian transcription by REV-ERB and ROR nuclear receptors. J. Biol. Rhythms 20, 391-403 (2005).

59. T. K. Sato et al., A functional genomics strategy reveals Rora as a component of the mammalian circadian clock. Neuron 43, 527-537 (2004).

60. S. M. Al Shareef et al., Kleine-Levin syndrome is associated with LMOD3 variants. J. Sleep Res. 28, e12718 (2019).

61. P. Cingolani et al., A program for annotating and predicting the effects of single nucleotide polymorphisms, SnpEff: SNPs in the genome of Drosophila melanogaster strain w1118; iso-2; iso-3. Fly (Austin) 6, 80-92 (2012).

62. U. A. Schatz et al., Evidence of mild founder LMOD3 mutations causing nemaline myopathy 10 in Germany and Austria. Neurology 91, e1690-e1694 (2018).

63. R. Tillman, B. Geller, Definitions of rapid, ultrarapid, and ultradian cycling and of episode duration in pediatric and adult bipolar disorders: A proposal to distinguish episodes from cycles. J. Child Adolesc. Psychopharmacol. 13, 267-271 (2003).

64. E. Groos et al., Emerging psychiatric disorders in Kleine-Levin syndrome. J. Sleep Res. 27, e12690 (2018).

65. K. A. Kaplan et al., Hypersomnia subtypes, sleep and relapse in bipolar disorder. Psychol. Med. 45, 1751-1763 (2015).

66. R. B. Grigolon et al., Hypersomnia and bipolar disorder: A systematic review and meta-analysis of proportion. J. Affect. Disord. 246, 659-666 (2019).

67. L. Pagani et al., Genetic contributions to circadian activity rhythm and sleep pattern phenotypes in pedigrees segregating for severe bipolar disorder. Proc. Natl. Acad. Sci. U.S.A. 113, E754-E761 (2016).

68. E. Bora, Neurocognitive features in clinical subgroups of bipolar disorder: A metaanalysis. J. Affect. Disord. 229, 125-134 (2018).

69. I. M. M. Lima, A. D. Peckham, S. L. Johnson, Cognitive deficits in bipolar disorders: Implications for emotion. Clin. Psychol. Rev. 59, 126-136 (2018).

70. J. M. Lane et al., Genome-wide association analyses of sleep disturbance traits identify new loci and highlight shared genetics with neuropsychiatric and metabolic traits. Nat. Genet. 49, 274-281 (2017).

71. K. J. S. Lewis et al., Comparison of genetic liability for sleep traits among individuals with bipolar disorder I or II and control participants. JAMA Psychiatry 77, 303-310 (2019).

72. M. Engström, F. Latini, A. M. Landtblom, Neuroimaging in the Kleine-Levin syndrome. Curr. Neurol. Neurosci. Rep. 18, 58 (2018).

73. S. B. Hong, Neuroimaging of narcolepsy and Kleine-Levin syndrome. Sleep Med. Clin 12, 359-368 (2017)

74. A. Kas, S. Lavault, M. O. Habert, I. Arnulf, Feeling unreal: A functional imaging study in patients with Kleine-Levin syndrome. Brain 137, 2077-2087 (2014).

75. C. Blume, C. Garbazza, M. Spitschan, Effects of light on human circadian rhythms, sleep and mood. Somnologie (Berl.) 23, 147-156 (2019).

76. C. A. Czeisler, J. C. Zimmerman, J. M. Ronda, M. C. Moore-Ede, E. D. Weitzman, Timing of REM sleep is coupled to the circadian rhythm of body temperature in man. Sleep 2, 329-346 (1980).

77. M. A. Carskadon, W. C. Dement, Sleep studies on a 90-minute day. Electroencephalogr. Clin. Neurophysiol. 39, 145-155 (1975).

78. A. Goldbart et al., Narcolepsy and predictors of positive MSLTs in the Wisconsin sleep cohort. Sleep (Basel) 37, 1043-1051 (2014).

79. L. Chen, Y.-S. Huang, "Actigraphy study and different hypersomnia disorders" in 2 nd Asian Narcolepsy \& Hypersomnolence Society Meeting (2019), vol. 2, p. 39.

80. A. K. Gold, G. Kinrys, Treating circadian rhythm disruption in bipolar disorder. Curr. Psychiatry Rep. 21, 14 (2019).

81. A. Vreeker et al., Genetic analysis of activity, brain and behavioral associations in extended families with heavy genetic loading for bipolar disorder. Pyschol. Med. $1-9,10.1017 /$ S0033291719003416 (2019).

82. M. Ikeda et al., A genome-wide association study identifies two novel susceptibility loci and trans population polygenicity associated with bipolar disorder. Mol. Psy chiatry 23, 639-647 (2018).

83. Psychiatric GWAS Consortium Bipolar Disorder Working Group, Large-scale genomewide association analysis of bipolar disorder identifies a new susceptibility locus near ODZ4. Nat. Genet. 43, 977-983 (2011).

84. A. J. Forstner et al., Identification of shared risk loci and pathways for bipolar disorder and schizophrenia. PLoS One 12, e0171595 (2017).

85. S. Ripke et al.; Multicenter Genetic Studies of Schizophrenia Consortium; Psychosis Endophenotypes International Consortium; Wellcome Trust Case Control Consortium 2, Genome-wide association analysis identifies 13 new risk loci for schizophrenia. Nat. Genet. 45, 1150-1159 (2013).

86. Schizophrenia Psychiatric Genome-Wide Association Study (GWAS) Consortium Genome-wide association study identifies five new schizophrenia loci. Nat. Genet. 43, 969-976 (2011).
87. J. Ward et al., Genome-wide analysis in UK Biobank identifies four loci associated with mood instability and genetic correlation with major depressive disorder, anxiety disorder and schizophrenia. Transl. Psychiatry 7, 1264 (2017).

88. G. Weber, Investigation of metal-species ( $\mathrm{Ca}, \mathrm{Mg}, \mathrm{Zn}, \mathrm{Fe}, \mathrm{Cu}, \mathrm{Pb}, \mathrm{Cd}, \mathrm{Sn})$ in urine by HPLC-AAS. J. Trace Elem. Electrolytes Health Dis. 2, 61-65 (1988).

89. K. J. Tsuchiya, M. Byrne, P. B. Mortensen, Risk factors in relation to an emergence of bipolar disorder: A systematic review. Bipolar Disord. 5, 231-242 (2003).

90. D. M. Lyall et al., Low birth weight and features of neuroticism and mood disorder in 83545 participants of the UK Biobank cohort. BJPsych Open 2, 38-44 (2016).

91. T. Kikas, K. Rull, R. N. Beaumont, R. M. Freathy, M. Laan, The effect of genetic variation on the placental transcriptome in humans. Front. Genet. 10, 550 (2019).

92. A. Brockenshire, The "mini-mental state": A handy tool. Perspectives 11, 7-8 (1987).

93. M. Horikoshi et al.; CHARGE Consortium Hematology Working Group; Early Growth Genetics (EGG) Consortium, Genome-wide associations for birth weight and correlations with adult disease. Nature 538, 248-252 (2016).

94. N. M. Warrington et al.; EGG Consortium, Maternal and fetal genetic effects on birth weight and their relevance to cardio-metabolic risk factors. Nat. Genet. 51, 804-814 (2019).

95. R. N. Beaumont et al.; Early Growth Genetics (EGG) Consortium, Genome-wide association study of offspring birth weight in 86577 women identifies five novel loci and highlights maternal genetic effects that are independent of fetal genetics. Hum. Mol. Genet. 27, 742-756 (2018).

96. G. Zhang et al., Genetic associations with gestational duration and spontaneous preterm birth. N. Engl. J. Med. 377, 1156-1167 (2017)

97. L. Srinivasan et al.; Eunice Kennedy Shriver National Institute of Child Health and Human Development Neonatal Research Network, Genome-wide association study of sepsis in extremely premature infants. Arch. Dis. Child. Fetal Neonatal Ed. 102, F439-F445 (2017)

98. J. R. Pasqualini, P. Mercat, N. Giambiagi, Histone acetylation decreased by estradiol in the MCF-7 human mammary cancer cell line. Breast Cancer Res. Treat. 14, 101-105 (1989).

99. X. Jiang et al., Sodium valproate rescues expression of TRANK1 in iPSC-derived neural cells that carry a genetic variant associated with serious mental illness. Mol. Psychiatry 24, 613-624 (2019).

100. S. Purcell et al., PLINK: A tool set for whole-genome association and populationbased linkage analyses. Am. J. Hum. Genet. 81, 559-575 (2007).

101. E. L. Nicolazzi, D. lamartino, J. L. Williams, AffyPipe: An open-source pipeline for Affymetrix Axiom genotyping workflow. Bioinformatics 30, 3118-3119 (2014).

102. A. Auton et al.; 1000 Genomes Project Consortium, A global reference for human genetic variation. Nature 526, 68-74 (2015).

103. O. Delaneau, J. Marchini, J. F. Zagury, A linear complexity phasing method for thousands of genomes. Nat. Methods 9, 179-181 (2011).

104. O. Delaneau, J. Marchini; 1000 Genomes Project Consortium; 1000 Genomes Project Consortium, Integrating sequence and array data to create an improved $1000 \mathrm{Ge}-$ nomes Project haplotype reference panel. Nat. Commun. 5, 3934 (2014).

105. B. N. Howie, P. Donnelly, J. Marchini, A flexible and accurate genotype imputation method for the next generation of genome-wide association studies. PLoS Genet. 5, e1000529 (2009).

106. J. Marchini, B. Howie, S. Myers, G. McVean, P. Donnelly, A new multipoint method for genome-wide association studies by imputation of genotypes. Nat. Genet. 39, 906-913 (2007)

107. K. Watanabe, E. Taskesen, A. van Bochoven, D. Posthuma, Functional mapping and annotation of genetic associations with FUMA. Nat. Commun. 8, 1826 (2017).

108. S. D. Turner, qqman: An R package for visualizing GWAS results using Q-Q and manhattan plots. bioRxiv [Preprint] (2014). https://doi.org/10.1101/005165 (Accessed 20 March 2019).

109. M. Arnold, J. Raffler, A. Pfeufer, K. Suhre, G. Kastenmüller, SNiPA: An interactive, genetic variant-centered annotation browser. Bioinformatics 31, 1334-1336 (2015).

110. F. Hormozdiari et al., Colocalization of GWAS and eQTL signals detects target genes. Am. J. Hum. Genet. 99, 1245-1260 (2016).

111. J. Zheng et al.; Early Genetics and Lifecourse Epidemiology (EAGLE) Eczema Consortium, LD hub: A centralized database and web interface to perform LD score regression that maximizes the potential of summary level GWAS data for SNP heritability and genetic correlation analysis. Bioinformatics 33, 272-279 (2017).

112. H. Li, R. Durbin, Fast and accurate short read alignment with Burrows-Wheeler transform. Bioinformatics 25, 1754-1760 (2009).

113. K. Wang, M. Li, H. Hakonarson, ANNOVAR: Functional annotation of genetic variants from high-throughput sequencing data. Nucleic Acids Res. 38, e164 (2010).

114. S. Lee, G. R. Abecasis, M. Boehnke, X. Lin, Rare-variant association analysis: Study designs and statistical tests. Am. J. Hum. Genet. 95, 5-23 (2014). 